\title{
The Treatment of Medication-Related Osteonecrosis of the Jaw (MRONJ): A Systematic Review with a Pooled Analysis of Only Surgery versus Combined Protocols
}

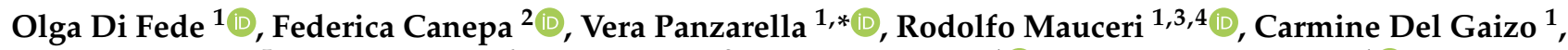 \\ Alberto Bedogni ${ }^{5}$, Vittorio Fusco ${ }^{6}$, Pietro Tozzo ${ }^{2}$, Giuseppe Pizzo ${ }^{1}{ }^{\circledR}$, Giuseppina Campisi ${ }^{1}(\mathbb{D}$ \\ and Antonio Galvano ${ }^{1}$ (D)
}

check for updates

Citation: Di Fede, O.; Canepa, F.; Panzarella, V.; Mauceri, R.; Del Gaizo, C.; Bedogni, A.; Fusco, V.; Tozzo, P.; Pizzo, G.; Campisi, G.; et al. The Treatment of Medication-Related Osteonecrosis of the Jaw (MRONJ): A Systematic Review with a Pooled Analysis of Only Surgery versus Combined Protocols. Int. J. Environ Res. Public Health 2021, 18, 8432. https://doi.org/10.3390/ ijerph18168432

Academic Editor: Paul B. Tchounwou

Received: 29 June 2021

Accepted: 3 August 2021

Published: 10 August 2021

Publisher's Note: MDPI stays neutral with regard to jurisdictional claims in published maps and institutional affiliations.

Copyright: (c) 2021 by the authors Licensee MDPI, Basel, Switzerland. This article is an open access article distributed under the terms and conditions of the Creative Commons Attribution (CC BY) license (https:/ / creativecommons.org/licenses/by/ $4.0 /)$.
1 Department of Surgical, Oncological and Oral Sciences (Di.Chir.On.S.), University of Palermo, 90127 Palermo, Italy; odifede@odonto.unipa.it (O.D.F.); rodolfo.mauceri@unipa.it (R.M.); delgaizocarmine@gmail.com (C.D.G.); giuseppe.pizzo@unipa.it (G.P.); campisi@odonto.unipa.it (G.C.); antonio.galvano@unipa.it (A.G.)

2 Operative Unit of Dentistry and Stomatology, Azienda Ospedaliera Ospedali Riuniti Villa Sofia Cervello, 90146 Palermo, Italy; canepafederica86@gmail.com (F.C.); tozzo.pietro@libero.it (P.T.)

3 Department of Biomedical and Dental Sciences and Morphofunctional Imaging, University of Messina, 98124 Messina, Italy

4 Department of Dental Surgery, Faculty of Dental Surgery, University of Malta, MSD 2090 Msida, Malta

5 Regional Center for Prevention, Diagnosis and Treatment of Medication and Radiation-Related Bone Diseases of the Head and Neck, University of Padova, 35128 Padova, Italy; alberto.bedogni@unipd.it

6 Azienda Ospedaliera SS. Antonio e Biagio e Cesare Arrigo, 15121 Alessandria, Italy; fusco.dott.vittorio@gmail.com

* Correspondence: panzarella@odonto.unipa.it; Tel./Fax: +39-091-6554615

\begin{abstract}
Medication-related osteonecrosis of the jaw (MRONJ) is a serious adverse reaction of antiresorptive and antiangiogenic agents, and it is also a potentially painful and debilitating condition. To date, no specific studies have prospectively evaluated the efficacy of its treatment and no robust standard of care has been established. Therefore, a systematic review (2007-2020) with a pooled analysis was performed in order to compare MRONJ surgical techniques (conservative or aggressive) versus combined surgical procedures (surgery plus a non-invasive procedure), where 1137 patients were included in the pooled analysis. A statistically significant difference in the 6-month improvement rate, comparing combined conservative surgery versus only aggressive ( $91 \%$ versus $72 \%, p=0.05)$, was observed. No significant difference regarding any group with respect to the 6-month total resolution rate $(82 \%$ versus $72 \%$ ) was demonstrated. Of note, conservative surgery combined with various, adjuvant, non-invasive procedures (ozone, LLLT or blood component + Nd:YAG) was found to achieve partial or full healing in all stages, with improved results and the amelioration of many variables. In conclusion, specific adjuvant treatments associated with minimally conservative surgery can be considered effective and safe in the treatment of MRONJ, although well-controlled studies are a requisite in arriving at definitive statements
\end{abstract}

Keywords: ONJ; osteonecrosis; treatment; therapy; surgery; staging

\section{Introduction}

Medication-related osteonecrosis of the jaw (MRONJ) is a drug-related adverse reaction, characterized by the progressive destruction of bone in the mandible or maxilla. It occurs in subjects currently or previously exposed to treatment with drugs in which an increased risk of osteonecrosis can be observed, in the absence of previous radiation treatment or metastatic disease to the jaw [1]. MRONJ is a severe, emerging orofacial disease, and thousands of cases have been reported since 2003 [2]. It can be associated with two categories of drugs: (1) anti-resorptive agents (e.g., bisphosphonates/BP and 
denosumab), used for the treatment of diseases involving the skeletal system in patients with bone metastases, hematological (e.g., myeloma) or osteometabolic disease (e.g., primary or secondary osteoporosis, Paget's disease), and (2) other non-antiresorptive drugs (antiangiogenic agents, mTOR inhibitors, monoclonal antibodies, etc.) for the treatment of different types of cancer. Recent data indicate that the incidence of MRONJ varies between 0 and 12.222 per 100,000 patient-years for cancer patients being treated with intravenous bisphosphonates. The incidence varies from 1.04 to 69 per 100,000 patient-years for osteometabolic patients when taking oral bisphosphonates, whereas this value is 30.2 per 100,000 patient-years [3,4] for patients taking denosumab. This variability depends mainly on several local and systemic risk factors. Regarding the latter, the most recognized are: primary disease in patients (cancer versus osteoporosis), a specific drug regimen, potency of the ONJ-related drug and the cumulative dose of BPs [1,5-7]. After a diagnosis of MRONJ, and whilst adhering to clinical and radiological protocols, the stage (i.e., severity and extent of the disease) must be defined in order to provide the patient with effective therapeutic management [7].

Whilst different treatments (therapeutic or palliative) have been described for MRONJ management, it is still a matter of controversy in the oral and maxillofacial communities that a gold standard has not yet been defined. In brief, this standard would involve the three main categories of MRONJ: (a) non-invasive procedures (ranging from pharmacological to laser treatment) [8,9], (b) invasive techniques (i.e., conservative or aggressive surgical approaches) [10] and (c) a combination of (a) and (b) (i.e., surgery plus one of the aforementioned non-invasive procedures) [11]. Non-invasive procedures include: medical treatment, intraoral vacuum-assisted treatment [12], the use of pentoxifylline (associated or not with tocopherol $[13,14])$, Er:YAG laser ablation, and Nd:YAG/diode laser biostimulation [15-17] and teriparatide [18-21]. Only partial and delayed healing has been reported with non-invasive techniques, to the exclusion of low-level laser treatment (LLLT) and, in certain cases, teriparatide. Furthermore, there is a paucity of high-impact studies in the literature, which would demonstrate effective positive outcomes [22].

Surgical treatments comprise: (i) conservative approaches, such as bone debridement, and sequestrectomy, and (ii) invasive, more aggressive procedures, such as re-sectioning the affected bone and jawbone reconstruction, where indicated. Several studies have yielded very positive results for surgical treatment in MRONJ treatment, especially if performed in the early stages of the disease [23-26].

Many in the field consider that the term 'treatment' is often used inappropriately, in that it is not possible for the disease to heal completely or for the majority of MRONJ patients to arrive at a state of remission. Thus, and as documented in the MRONJ literature, treatment goals are mainly concerned with managing pain, controlling for any infection of the soft and hard tissues and reducing the progression or occurrence of bone necrosis [11]. Over and above every consideration, the authors of this paper hold that maximizing a patient's quality of life has to be a key feature of every protocol requiring MRONJ treatment.

The aim of this systematic review with a pooled analysis is to examine and compare the main categories of MRONJ treatment: surgical techniques (conservative or aggressive) versus combined procedures (surgery plus non-invasive procedures), by focusing on their therapeutic effectiveness. The recommendations outlined by the Prisma-P 2015 checklist systematic review protocol were followed in order to formulate the methodology for this systematic review.

\section{Materials and Methods}

The present study followed the Preferred Reporting Items for Systematic Reviews and Meta-Analyses (PRISMA) and the Meta-analysis of Observational Studies in Epidemiology (MOOSE) guidelines. The PICO search strategy considered: patients who underwent conservative or aggressive surgery with or without combined procedures (auxiliary treatments). Selected outcomes were a 6-month complete resolution rate, or a 6-month improvement rate in terms of a transition from a higher to a lower stage. 


\subsection{Eligibility Criteria}

In order to be included in the systematic review outlined in this paper, studies had to include results from: prospective, non-randomized and randomized clinical trials, retrospective cohort studies and case series $(n \geq 10)$, which investigated the role of surgical (conservative or aggressive) techniques with or without combined procedures (surgery plus a non-invasive one) and with a follow-up $\geq 6$ months. Studies were excluded if they constituted a Commentary, Review, Editorial or Protocol. Case series $(n<10)$ or case reports were excluded from the pooled analysis, and the studies were limited to research regarding human beings.

\subsection{Information Sources and Search Strategy}

A systematic, electronic search through different biomedical databases (e.g., PubMed, Ovide/MEDLINE, Web of Knowledge, Embase and the Cochrane Library) was performed by two authors (A.G. and F.C.) in the period from January 2007 to December 2020, and it was restricted to abstracts in English. The MeSH searched terms are included in Table 1.

Table 1. MeSH terms.

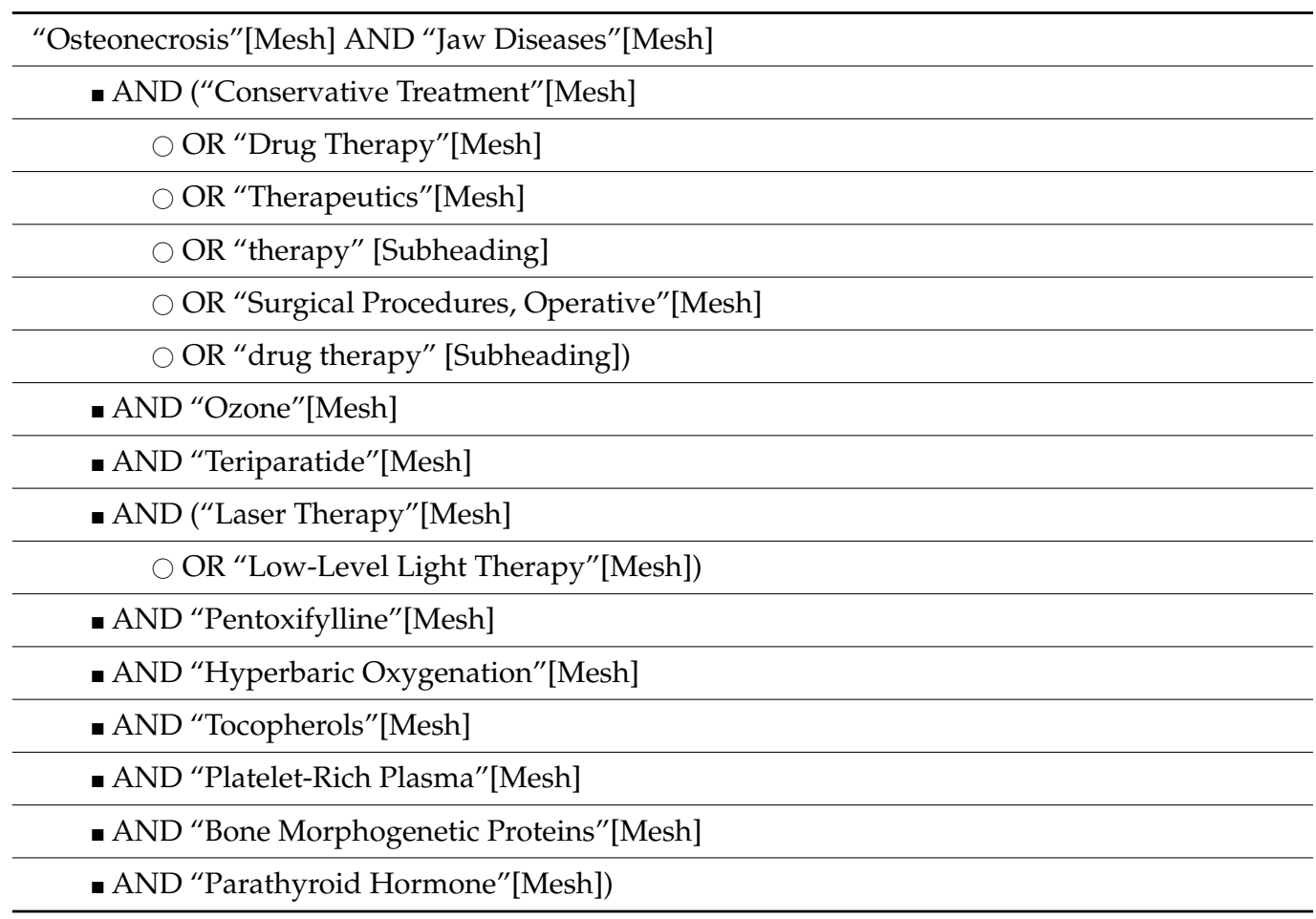

Furthermore, other data sources (from international meetings and indexed dentistry journals such as Journal of Dentistry, Journal of Oral Maxillofacial Surgery, Journal of Dental Research) were scanned as a source of grey literature.

\subsubsection{Study Selection}

Screening and eligibility were assessed independently by two reviewers (F.C. and O.D.F.), who were in agreement regarding the results. The Titles of papers and Abstracts were initially screened for relevance and possible eligible results, and thereafter full texts were retrieved. Finally, the reviewers combined their results to create a corpus of selected papers to assess for final eligibility. According to the aim of this review, the resulting papers were allocated to four experimental categories: (1) conservative surgery, (2) aggressive surgery, (3) a conservative plus non-invasive procedure and (4) aggressive surgery plus non-invasive protocols. Tables 2 and 3 summarize the eligible studies. 
Table 2. Summary of the characteristics and the results of the studies concerning MRONJ surgical therapies.

\begin{tabular}{|c|c|c|c|c|c|c|}
\hline Treatment & Study & Study Type & Pts & Intervention & Outcome & Follow-Up \\
\hline \multirow{8}{*}{$\begin{array}{l}\text { Conservative } \\
\text { Surgery }\end{array}$} & $\begin{array}{l}\text { De Souza Povoa } \\
\text { et al., } 2016\end{array}$ & Case report & $\begin{array}{c}\mathrm{N}=1 \\
\text { Onc } \\
\text { Stage } 1\end{array}$ & $\begin{array}{l}\text { Removal of the exposed necrotic bone and primary } \\
\text { wound closure }\end{array}$ & $\begin{array}{l}\text { Complete healing and } \\
\text { new bone formation in } \\
\text { the surgical site }\end{array}$ & 26 months \\
\hline & Ribeiro et al., 2015 & Case report & $\begin{array}{l}\qquad \mathrm{N}=1 \\
\text { Ost } \\
\text { Stage unspecified }\end{array}$ & $\begin{array}{l}\text { Surgical removal of whole necrotic bone, extraction of } \\
\text { all compromised teeth }\end{array}$ & Complete healing & 12 months \\
\hline & $\begin{array}{l}\text { De Souza Faloni } \\
\text { et al., } 2011\end{array}$ & Case report & $\begin{array}{c}\mathrm{N}=1 \\
\text { Ost } \\
\text { Stage } 2\end{array}$ & $\begin{array}{l}\text { Conservative debridement of the necrotic bone and of } \\
\text { part of the surrounding healthy bone, as a margin of } \\
\text { safety }\end{array}$ & Complete healing & 8 months \\
\hline & $\begin{array}{l}\text { Pechalova et al., } \\
\qquad 2011\end{array}$ & Case series & $\begin{array}{c}\mathrm{N}=3 \\
\text { Onc } \\
\text { Stage unspecified }\end{array}$ & Conservative surgical debridement & Complete healing & $\begin{array}{c}\text { Average of } 4 \\
\text { months }\end{array}$ \\
\hline & Martins et al., 2012 & $\begin{array}{l}\text { Retrospective } \\
\text { clinical study }\end{array}$ & $\begin{array}{c}\mathrm{N}=5 \\
\text { Onc } \\
\text { Stage } 1,2\end{array}$ & $\begin{array}{l}\text { Sequestrectomy and/or ostectomy and/or osteoplasty } \\
\text { until bone marrow bleeding }\end{array}$ & $\begin{array}{l}60 \% \text { patients completely } \\
\text { healed }\end{array}$ & 6 months \\
\hline & Jung et al., 2017 & Case series & $\begin{array}{c}\mathrm{N}=7 \\
\text { Ost } \\
\text { Stage } 2,3\end{array}$ & $\begin{array}{l}\text { Patient underwent conventional surgery, and the bone } \\
\text { defects were filled with absorbable collagen plugs. }\end{array}$ & $\begin{array}{l}\text { Complete healing and } \\
\text { new bone formation in } \\
\text { the surgical site }\end{array}$ & 3 months \\
\hline & Atalay et al., 2011 & $\begin{array}{l}\text { Retrospective } \\
\text { clinical study }\end{array}$ & $\begin{array}{l}\mathrm{N}=10 \\
\text { Onc } \\
\text { Stage }\end{array}$ & $\begin{array}{l}\text { The affected bony tissues were curetted from the } \\
\text { surface of the bone using bone curettes and round } \\
\text { tungsten carbide burs. The necrotic bone was } \\
\text { completely removed until the vital bone tissues and } \\
\text { vessel spots appeared }\end{array}$ & $\begin{array}{l}40 \% \text { patients completely } \\
\text { healed }\end{array}$ & 6 months \\
\hline & Vescovi et al., 2012 & $\begin{array}{l}\text { Retrospective } \\
\text { clinical study }\end{array}$ & $\begin{array}{c}\mathrm{N}=17 \\
\text { Onc + Ost } \\
\text { Stage } 1,2,3\end{array}$ & $\begin{array}{l}\text { Conservative surgical treatments consisted of } \\
\text { sequestrectomy of necrotic bone, superficial } \\
\text { debridement/curettage, or corticotomy/surgical } \\
\text { removal of alveolar and/or cortical bone }\end{array}$ & $\begin{array}{l}53 \% \text { patients completely } \\
\text { healed }\end{array}$ & 9 months \\
\hline
\end{tabular}


Table 2. Cont.

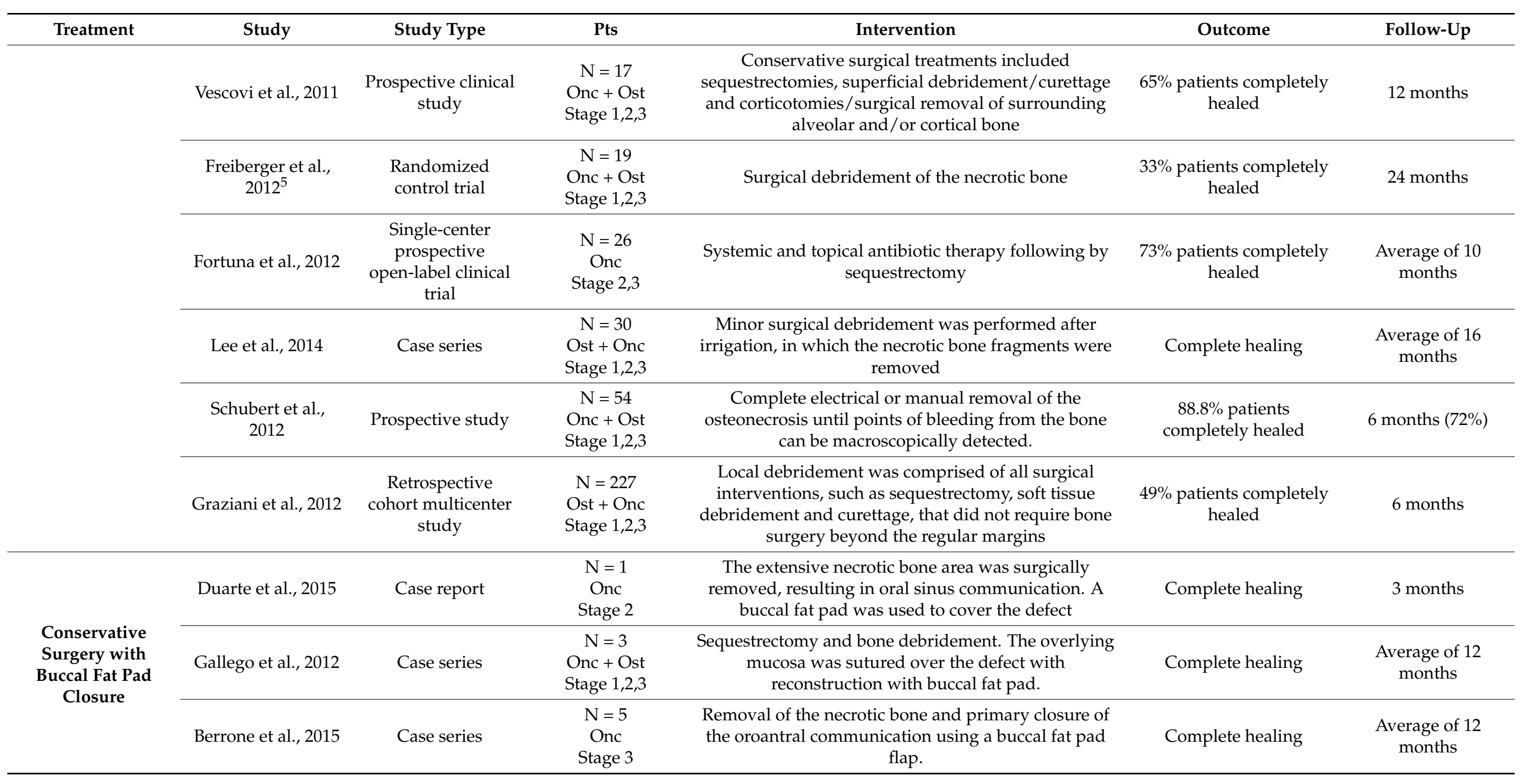


Table 2. Cont.

\begin{tabular}{|c|c|c|c|c|c|c|}
\hline Treatment & Study & Study Type & Pts & Intervention & Outcome & Follow-Up \\
\hline & Lopes et al., 2015 & $\begin{array}{l}\text { Retrospective } \\
\text { observational } \\
\text { cohort study }\end{array}$ & $\begin{array}{c}\mathrm{N}=46 \\
\text { Onc + Ost } \\
\text { Stage } 2,3\end{array}$ & $\begin{array}{c}\text { Removal of all necrotic bone until bleeding was } \\
\text { obtaining at the bony margins, conscious smoothing of } \\
\text { all sharp bone edges and primary closure of the } \\
\text { wound. }\end{array}$ & $\begin{array}{l}87 \% \text { patients } \\
\text { completely healed }\end{array}$ & 10 months \\
\hline & $\begin{array}{l}\text { Hayashida et al., } \\
2017\end{array}$ & $\begin{array}{l}\text { Multicenter } \\
\text { retrospective study }\end{array}$ & $\begin{array}{c}\mathrm{N}=38 \\
\text { Onc + Ost } \\
\text { Stage } 1,2,3\end{array}$ & $\begin{array}{l}\text { One group received conservative surgery, removal of } \\
\text { only the necrotic bone and extensive surgery, defined } \\
\text { as removal of the necrotic and surrounding bone } \\
\text { (marginal mandibulectomy or partial maxillectomy). }\end{array}$ & $\begin{array}{l}76.7 \% \text { patients } \\
\text { completely healed }\end{array}$ & $\begin{array}{l}\text { Average of } 15 \\
\text { months }\end{array}$ \\
\hline \multirow{7}{*}{$\begin{array}{l}\text { Aggressive } \\
\text { Surgery }\end{array}$} & Hewson et al., 2012 & Case report & $\begin{array}{c}\mathrm{N}=1 \\
\text { Onc } \\
\text { Stage } 3\end{array}$ & $\begin{array}{l}\text { Radical surgical excision of all diseased bone and } \\
\text { nasio-labial flap reconstruction. }\end{array}$ & Complete healing & 6 months \\
\hline & Ghazali et al., 2013 & Case report & $\begin{array}{c}\mathrm{N}=1 \\
\text { Ost } \\
\text { Stage } 3\end{array}$ & $\begin{array}{l}\text { Hemimandibulectomy and an osteocutaneous fibula } \\
\text { flap reconstruction }\end{array}$ & Complete healing & 24 months \\
\hline & Shintani et al., 2015 & Cohort study & $\begin{array}{c}\mathrm{N}=4 \\
\text { Ost + Onc } \\
\text { Stage } 1,2,3\end{array}$ & $\begin{array}{l}\text { Segmental resection and immediate reconstruction } \\
\text { with a reconstruction plate were performed. }\end{array}$ & $\begin{array}{l}3 / 4 \text { patients } \\
\text { completely healed }\end{array}$ & 12 months \\
\hline & Lee et al., 2014 & Case report & $\begin{array}{c}\mathrm{N}=10 \\
\text { Ost }+ \text { Onc } \\
\text { Stage } 1,2,3\end{array}$ & $\begin{array}{l}\text { Large necrotic bone segment was removed by an } \\
\text { ultrasonic bone saw. A bone file or rongeur was used } \\
\text { for rounding the sharp bone edge. Then, the bone } \\
\text { defect was closed by sutures or COE pack. }\end{array}$ & Complete healing & $\begin{array}{l}\text { Average of } 8 \\
\text { months }\end{array}$ \\
\hline & $\begin{array}{l}\text { Hanasono et al., } \\
2013\end{array}$ & Case series & $\begin{array}{c}\mathrm{N}=13 \\
\text { Onc } \\
\text { Stage2, } 3\end{array}$ & $\begin{array}{l}\text { Segmental mandibulectomy and microvascular free } \\
\text { flap reconstruction. }\end{array}$ & Complete healing & $\begin{array}{l}\text { Average of } 15 \\
\text { months }\end{array}$ \\
\hline & Graziani et al., 2012 & $\begin{array}{l}\text { Retrospective } \\
\text { cohort multicenter } \\
\text { study }\end{array}$ & $\begin{array}{l}\mathrm{N}=120 \\
\text { Ost + Onc } \\
\text { Stage } 1,2,3\end{array}$ & $\begin{array}{c}\text { Re-sective procedures were defined as corticotomy, } \\
\text { surgical removal of the lesion and extended bone } \\
\text { removal without prejudice for the continuity of the } \\
\text { mandible/maxilla. }\end{array}$ & $\begin{array}{l}68 \% \text { patients } \\
\text { completely healed }\end{array}$ & 6 months \\
\hline & $\begin{array}{l}\text { Hayashida et al., } \\
2017\end{array}$ & $\begin{array}{l}\text { Multicenter } \\
\text { retrospective study }\end{array}$ & $\begin{array}{c}\mathrm{N}=121 \\
\text { Onc + Ost } \\
\text { Stage } 1,2,3\end{array}$ & $\begin{array}{l}\text { Extensive surgery, defined as removal of the necrotic } \\
\text { and surrounding bone (marginal mandibulectomy or } \\
\text { partial maxillectomy). }\end{array}$ & $\begin{array}{l}86.8 \% \text { patients } \\
\text { completely healed }\end{array}$ & $\begin{array}{c}\text { Average of } 15 \\
\text { months }\end{array}$ \\
\hline
\end{tabular}


Table 3. Summary of the characteristics and the results of the studies on MRONJ surgery plus non-invasive procedures.

\begin{tabular}{|c|c|c|c|c|c|c|}
\hline & Study & Study Type & Population & Intervention & Outcome & Follow-Up \\
\hline \multicolumn{7}{|c|}{ Conservative surgery plus $(+)$ non-invasive procedures } \\
\hline \multirow{8}{*}{$\begin{array}{l}\text { 1. Surgery + Blood } \\
\text { Component }\end{array}$} & Gönen et al., 2017 & Case report & $\begin{array}{c}\mathrm{N}=1 \\
\text { Onc } \\
\text { Stage } 3\end{array}$ & Sequestrectomy + PRF & Complete resolution & 18 months \\
\hline & Soydan et al., 2014 & Case report & $\begin{array}{c}\mathrm{N}=1 \\
\text { Onc } \\
\text { Stage unspecified }\end{array}$ & Curettage + PRF & Complete resolution & 6 months \\
\hline & Maluf et al., 2016 & Case series & $\begin{array}{c}\mathrm{N}=2 \\
\text { Onc } \\
\text { Stage } 2\end{array}$ & $\begin{array}{l}\text { Resection of the necrotic tissues, } \\
\text { curettage and osteotomy + L-PRF }\end{array}$ & Partial healing & 6 months \\
\hline & Dincă et al., 2014 & $\begin{array}{l}\text { Retrospective clinical } \\
\text { study }\end{array}$ & $\begin{array}{c}\mathrm{N}=10 \\
\text { Onc } \\
\text { Stage } 2\end{array}$ & Sequestrectomy or curettage $+\mathrm{PRF}$ & Complete resolution & 1 month \\
\hline & Nørholt et al., 2016 & Prospective study & $\begin{array}{c}\mathrm{N}=15 \\
\text { Onc + Ost } \\
\text { Stage 2,3 }\end{array}$ & Curettage + L-PRF & $\begin{array}{l}93.3 \% \text { patients } \\
\text { completely healed }\end{array}$ & 20 months \\
\hline & $\begin{array}{l}\text { Bocanegra-Pérez } \\
\text { et al., } 2012\end{array}$ & $\begin{array}{c}\text { Prospective } \\
\text { descriptive study }\end{array}$ & $\begin{array}{c}\mathrm{N}=8 \\
\text { Onc + Ost } \\
\text { Stage } 2\end{array}$ & Curettage + PRP & Complete resolution & 14 months \\
\hline & Mozzati et al., 2012 & $\begin{array}{l}\text { Retrospective clinical } \\
\text { study }\end{array}$ & $\begin{array}{c}\mathrm{N}=32 \\
\text { Onc } \\
\text { Stage } 2\end{array}$ & Conservative surgery + PRFG & Complete resolution & From 48 to 50 months \\
\hline & Tsai et al., 2016 & Case report & $\begin{array}{c}\mathrm{N}=1 \\
\text { Ost } \\
\text { Stage } 3\end{array}$ & $\begin{array}{l}\text { Surgical debridement, } \\
\text { sequestrectomy + PRF }\end{array}$ & Complete resolution & 10 months \\
\hline
\end{tabular}


Table 3. Cont.

\begin{tabular}{|c|c|c|c|c|c|c|}
\hline & Study & Study Type & Population & Intervention & Outcome & Follow-Up \\
\hline & Pelaz et al., 2014 & Cohort study & $\begin{array}{c}\mathrm{N}=5 \\
\text { Ost } \\
\text { Stage } 3\end{array}$ & $\begin{array}{c}\text { Sequestrectomy and curettage }+ \\
\text { PRF }\end{array}$ & Complete resolution & $\begin{array}{l}\text { An average of } 20 \\
\text { months }\end{array}$ \\
\hline & Park et al., 2017 & Prospective study & $\begin{array}{c}\mathrm{N}=25 \\
\text { Onc + Ost } \\
\text { Stage } 1,2,3\end{array}$ & Conservative surgery + L-PRF & $\begin{array}{c}36 \% \text { patients } \\
\text { completely healed }\end{array}$ & 4 months \\
\hline & $\begin{array}{c}\text { Fernando de } \\
\text { Almeida Barros } \\
\text { Mourao C et al., } 2020\end{array}$ & Case series & $\begin{array}{c}\mathrm{N}=11 \\
\text { Ost } \\
\text { Stage } 2\end{array}$ & $\begin{array}{l}\text { Surgical removal of necrotic bone }+ \\
\text { PRF membranes }\end{array}$ & Complete healing & 24 months \\
\hline & Giudice A et al., 2020 & Case report & $\begin{array}{c}\mathrm{N}=1 \\
\text { Ost } \\
\text { Stage } 3\end{array}$ & $\begin{array}{l}\text { Surgical removal of necrotic bone }+ \\
\text { PRF membranes }\end{array}$ & Complete healing & 60 months \\
\hline & Bouland C et al., 2020 & Case report & $\begin{array}{c}\mathrm{N}=2 \\
\text { Ost }+ \text { Onc } \\
\text { Stage } 2 \text { and } 3\end{array}$ & $\begin{array}{l}\text { Surgical removal of necrotic bone + } \\
\text { SVF and L-PRF membranes }\end{array}$ & Complete healing & 18 months \\
\hline $\begin{array}{c}\text { 2. Surgery + Blood } \\
\text { Component }+ \\
\text { Photodynamic Therapy }\end{array}$ & De Castro et al., 2016 & Case series & $\begin{array}{c}\mathrm{N}=2 \\
\text { Ost } \\
\text { Stage } 2,3\end{array}$ & Surgical debridement + PDT + PRF & Complete resolution & $\begin{array}{l}\text { An average of } 12 \\
\text { months }\end{array}$ \\
\hline $\begin{array}{l}\text { 3. Surgery }+ \text { Blood } \\
\text { Component }+ \\
\text { Bone Morphogenetic } \\
\text { Protein }\end{array}$ & Park et al., 2017 & Prospective study & $\begin{array}{c}\mathrm{N}=30 \\
\text { Onc + Ost } \\
\text { Stage } 1,2,3\end{array}$ & $\begin{array}{l}\text { Conservative surgery }+ \text { combined } \\
\text { L-PRF and recombinant human } \\
\text { BMP-2 (rhBMP-2) }\end{array}$ & $\begin{array}{c}60 \% \text { patients } \\
\text { completely healed }\end{array}$ & 4 months \\
\hline 4. Surgery + Teriparatide & Lee et al., 2010 & Case report & $\begin{array}{c}\mathrm{N}=1 \\
\text { Ost } \\
\text { Stage } 2\end{array}$ & Sequestrectomy + teriparatide & Complete resolution & 6 months \\
\hline $\begin{array}{l}\text { 5. Surgery + Teriparatide }+ \\
\text { Bone Morphogenetic } \\
\text { Protein }\end{array}$ & Jung et al., 2017 & Cohort study & $\begin{array}{c}\mathrm{N}=6 \\
\text { Ost } \\
\text { Stage } 2,3\end{array}$ & $\begin{array}{l}\text { Conservative surgery and } \\
\text { absorbable collagen plugs soaked } \\
\text { by rhBMP-2 into the bone defect } \\
\text { plus daily subcutaneous injection of } \\
20 \text { mg teriparatide for } 1-4 \text { months. }\end{array}$ & Complete resolution & 3 months \\
\hline
\end{tabular}


Table 3. Cont.

\begin{tabular}{|c|c|c|c|c|c|c|}
\hline & Study & Study Type & Population & Intervention & Outcome & Follow-Up \\
\hline $\begin{array}{l}\text { 6. Surgery + Bone } \\
\text { Morphogenetic Protein }\end{array}$ & Jung et al., 2017 & Cohort study & $\begin{array}{c}\mathrm{N}=4 \\
\text { Ost } \\
\text { Stage } 2,3\end{array}$ & $\begin{array}{l}\text { Conservative surgery and } \\
\text { absorbable collagen plugs soaked } \\
\text { by rhBMP-2 into the bone defect. }\end{array}$ & Complete resolution & 3 months \\
\hline $\begin{array}{c}\text { 7. Surgery }+ \text { Blood } \\
\text { Component }+ \text { Autolugus } \\
\text { Bone Marrow Stem Cells }\end{array}$ & $\begin{array}{l}\text { Gonzálvez-García } \\
\text { et al., } 2013\end{array}$ & Case report & $\begin{array}{c}\mathrm{N}=1 \\
\text { Onc } \\
\text { Stage } 2\end{array}$ & $\begin{array}{l}\text { Removal of the necrotic bone+ bone } \\
\text { marrow stem cells + beta tricalcium } \\
\text { phosphate + demineralized bone } \\
\text { matrix + PRP }\end{array}$ & Complete resolution & 6 months \\
\hline 8. Surgery + LLLT & $\begin{array}{l}\text { Da Guarda et al., } \\
2012\end{array}$ & Case report & $\begin{array}{c}\mathrm{N}=1 \\
\text { Onc } \\
\text { Stage unspecified }\end{array}$ & $\begin{array}{c}\text { GaAlAs diode laser every } 48 \mathrm{~h} \text { for } \\
10 \text { days + antibiotic therapy + } \\
\text { curettage }\end{array}$ & Complete resolution & 6 months \\
\hline \multirow{5}{*}{$\begin{array}{l}\text { 9. Surgery + Blood } \\
\text { Component + Laser } \\
\text { Phototherapy }\end{array}$} & $\begin{array}{l}\text { Altay et al., } \\
2014\end{array}$ & $\begin{array}{l}\text { Retrospective clinical } \\
\text { study }\end{array}$ & $\begin{array}{c}\mathrm{N}=11 \\
\text { Onc } \\
\text { Stage2,3 }\end{array}$ & $\begin{array}{l}\text { Pre- and post-operative antibiotic } \\
\text { administrations + GaA-lAs diode } \\
\text { laser }\end{array}$ & Complete resolution & 12 months \\
\hline & $\begin{array}{l}\text { Atalay et al., } \\
2011\end{array}$ & $\begin{array}{l}\text { Retrospective clinical } \\
\text { study }\end{array}$ & $\begin{array}{l}\mathrm{N}=10 \\
\text { Onc } \\
\text { Stage } 1,2\end{array}$ & $\begin{array}{c}\text { Conservative surgery + low-level } \\
\text { laser therapy application (Er:YAG } \\
\text { and Nd:YAG) }\end{array}$ & $\begin{array}{l}70 \% \text { patients } \\
\text { completely healed }\end{array}$ & 12 months \\
\hline & Vescovi et al., 2012 & $\begin{array}{l}\text { Retrospective clinical } \\
\text { study }\end{array}$ & $\begin{array}{c}\mathrm{N}=45 \\
\text { Onc + Ost } \\
\text { Stage } 1,2,3\end{array}$ & $\begin{array}{c}\text { Conservative surgery + laser } \\
\text { Nd:YAG }\end{array}$ & $\begin{array}{c}89 \% \text { patients } \\
\text { completely healed }\end{array}$ & 6 months \\
\hline & $\begin{array}{l}\text { Vescovi et al., } \\
2011\end{array}$ & $\begin{array}{c}\text { Prospective clinical } \\
\text { study }\end{array}$ & $\begin{array}{c}\mathrm{N}=62 \\
\text { Onc + Ost } \\
\text { Stage } 1,2,3\end{array}$ & Conservative surgery + laser LLLT & $\begin{array}{l}73 \% \text { patients } \\
\text { completely healed }\end{array}$ & 17 months. \\
\hline & $\begin{array}{l}\text { Martins et al., } \\
\quad 2012\end{array}$ & $\begin{array}{l}\text { Retrospective clinical } \\
\text { study }\end{array}$ & $\begin{array}{c}\mathrm{N}=14 \\
\text { Onc } \\
\text { Stage } 1,2,3\end{array}$ & $\begin{array}{l}\text { Conservative surgery }+ \text { continuous } \\
\text { indium-gallium-aluminum- } \\
\text { phosphide diode laser. The LPT } \\
\text { treatment started on the first visit } \\
\text { and continued daily until mucosal } \\
\text { healing was observed. }\end{array}$ & $\begin{array}{c}86 \% \text { patients } \\
\text { completely healed }\end{array}$ & 12 months \\
\hline
\end{tabular}


Table 3. Cont.

\begin{tabular}{|c|c|c|c|c|c|c|}
\hline & Study & Study Type & Population & Intervention & Outcome & Follow-Up \\
\hline 10. Surgery + Ozone & $\begin{array}{l}\text { Agrillo et al., } \\
\quad 2012\end{array}$ & Retrospective study & $\begin{array}{c}\mathrm{N}=94 \\
\text { Onc + Ost } \\
\text { Stage unspecified }\end{array}$ & $\begin{array}{c}\text { Curettage or sequestrectomy }+ \\
\text { Ozone therapy (3 min sessions } \\
\text { 2/week) + pharmacological therapy }\end{array}$ & $\begin{array}{c}90 \% \text { patients } \\
\text { completely healed }\end{array}$ & $\begin{array}{c}\text { An average of } 6 \\
\text { months }\end{array}$ \\
\hline \multirow{3}{*}{ 11. $\mathrm{HBO}+$ Surgery * } & $\begin{array}{l}\text { Fatema et al., } \\
2013\end{array}$ & Case report & $\begin{array}{c}\mathrm{N}=1 \\
\text { Onc } \\
\text { Stage } 2\end{array}$ & $\begin{array}{l}\text { Antibiotics therapy, irrigation, } \\
\text { pre-operative HBO therapy for } 20 \\
\text { sessions, conservative minor } \\
\text { surgical debridement and again } \\
\text { post-operative HBO therapy for ten } \\
\text { sessions. }\end{array}$ & Complete resolution & Unspecified \\
\hline & $\begin{array}{l}\text { Al-Zoman et al., } \\
2013\end{array}$ & Case series & $\begin{array}{c}\mathrm{N}=3 \\
\text { Onc } \\
\text { Stage2,3 }\end{array}$ & $\begin{array}{l}\text { HBO therapy, oral/parenteral } \\
\text { antibiotic, analgesics, conservative } \\
\text { surgery (debridement of bone } \\
\text { sequestra) and daily rinsing with } \\
\text { chlorhexidine mouthwash. }\end{array}$ & Complete resolution & 12 months \\
\hline & Freiberger et al., 2012 & $\begin{array}{c}\text { Randomized control } \\
\text { trial }\end{array}$ & $\begin{array}{c}\mathrm{N}=24 \\
\text { Onc + Ost } \\
\text { Stage } 1,2,3\end{array}$ & $\begin{array}{l}40 \mathrm{HBO} \text { treatments at } 2.0 \text { atm for } 2 \mathrm{~h} \\
\text { twice per day and conservative } \\
\text { surgical debridement of the necrotic } \\
\text { bone. }\end{array}$ & $\begin{array}{c}52 \% \text { patients } \\
\text { completely healed }\end{array}$ & 24 months \\
\hline \multirow[b]{2}{*}{ 12. Ozone + Surgery* } & Ripamonti et al., 2012 & Case report & $\begin{array}{c}\mathrm{N}=1 \\
\text { Onc } \\
\text { Stage unspecified }\end{array}$ & $\begin{array}{l}\text { Antibiotic + antimycotic therapy for } \\
10 \text { days. Local ozone gas (total of } 15 \\
\text { applications). Conservative surgery } \\
\text { (sequestrectomy). }\end{array}$ & Complete resolution & 36 months \\
\hline & Brozoski et al., 2020 & Case series & $\begin{array}{l}\mathrm{N}=2 \\
\text { Onc }+ \text { Ost } \\
\text { Stage } 2\end{array}$ & $\begin{array}{l}\text { Weekly irrigation with aqueous } \\
\text { ozone solution on bone-exposed } \\
\text { region + daily mouthwashes of } \\
\text { ozone solution. After } 3 \text { and } 6 \\
\text { months: conservative surgery } \\
\text { (debridement and sequestrectomy) }\end{array}$ & Complete resolution & $\begin{array}{c}\text { An average of } 24 \\
\text { months }\end{array}$ \\
\hline
\end{tabular}


Table 3. Cont.

\begin{tabular}{|c|c|c|c|c|c|c|}
\hline & Study & Study Type & Population & Intervention & Outcome & Follow-Up \\
\hline \multirow[t]{3}{*}{ 13. Teriparatide + Surgery * } & Doh et al., 2015 & Case report & $\begin{array}{c}\mathrm{N}=1 \\
\text { Ost } \\
\text { Stage } 2\end{array}$ & $\begin{array}{l}\text { After } 4 \text { months of daily teriparatide } \\
\text { therapy conservative surgery } \\
\text { (sequestrectomy). The TPTD } \\
\text { therapy was terminated } 6 \text { months } \\
\text { after the initial treatment. }\end{array}$ & Complete resolution & 20 months \\
\hline & Kwon et al., 2012 & Case series & $\begin{array}{c}\mathrm{N}=6 \\
\text { Ost } \\
\text { Stage } 2,3\end{array}$ & $\begin{array}{l}\text { Daily Teriparatide ( } 20 \mu \mathrm{g} / \text { day) } 1-3 \\
\text { months }+ \text { conservative sequestrec- } \\
\text { tomy/marginal/aggressive } \\
\text { segmental resection }\end{array}$ & Complete resolution & 3 months \\
\hline & Kakehashi et al., 2015 & Case series & $\begin{array}{l}\mathrm{N}=10 \\
\text { Ost } \\
\text { Stage } 2,3\end{array}$ & $\begin{array}{l}\text { Daily teriparatide }(20 \mu \mathrm{g} / \text { day }) \\
\text { ranged from } 4 \text { to } 24 \text { months. In } \\
\text { some cases, surgery was performed } \\
\text { to obtain the healing. }\end{array}$ & Partial resolution & $\begin{array}{l}\text { From } 4 \text { to } 24 \text { months } \\
\text { (duration of } \\
\text { teriparatide therapy } \\
\text { until mucosal } \\
\text { healing) }\end{array}$ \\
\hline \multicolumn{7}{|c|}{ Aggressive surgery plus non-invasive procedures } \\
\hline $\begin{array}{l}\text { 1. Surgery + Bone Graft }+ \\
\text { Bone Morphogenetic } \\
\text { Protein }\end{array}$ & $\begin{array}{l}\text { Rahim I } \\
2015\end{array}$ & Case report & $\begin{array}{c}\mathrm{N}=1 \\
\text { Ost } \\
\text { Stage } 3\end{array}$ & $\begin{array}{l}\text { Partial mandibulectomy + bone } \\
\text { graft from the iliac crest + rhBMP-7 }\end{array}$ & Complete resolution & 60 months \\
\hline $\begin{array}{l}\text { 2. AF-Guided Surgery + } \\
\text { LLLT }\end{array}$ & $\begin{array}{l}\text { Vescovi P } \\
2015\end{array}$ & Case report & $\begin{array}{c}\mathrm{N}=1 \\
\text { Onc } \\
\text { Stage } 3\end{array}$ & $\begin{array}{l}\text { Osteotomy with Er:YAG laser + AF } \\
\text { visualization to guide the } \\
\text { osteoplasty. Intraoral irrigations } \\
\text { with povidone iodine solution + } \\
\text { application of Nd:YAG laser + } \\
\text { weekly applications of LLLT for } 3 \\
\text { weeks after intervention }\end{array}$ & Complete resolution & 7 months \\
\hline
\end{tabular}




\subsubsection{Data Collection Process}

Data collection was independently performed by two authors (F.C. and A.G.), and their results were reviewed by a third author (O.D.F.) to check for accuracy.

\subsubsection{Statistical Analysis}

Selected studies were reviewed to detect outcomes of interest. A standard extraction template was used to collect raw data. The extracted data comprised the sample size $(n)$, and treatment and clinical outcomes, including: (a) a 6-month complete resolution rate, or (b) a 6-month improvement (in terms of a transition from a higher to a lower stage) rate. Some results were not present in all the trials included in the review. The pooled results for each of the four categories were based on weighted estimates. The I2 statistic test was conducted in order to assess heterogeneity among the included studies. If an I-squared value was lower than 50\%, the fixed-effect-based Mantel-Haenszel model was used in order to present variables as weighted measures; if it was higher than $50 \%$, the pooled analysis was performed using the random effect-based model by Der Simonian and Laird. The overall (a) and (b) rates were reported as pooled proportions of the percentage of patients who had been treated with one of the four experimental categories. A twotailed Student's t-test with a significance level of 0.05 was used for all comparisons. All analyses were performed using the 15.0 MedCalc for Windows version (MedCalc Software, Ostend, Belgium).

\section{Results}

The initial search strategy identified 371 records, which were obtained by database searching, after the duplicates had been removed. Two reviewers (F.C. and A.G.) independently screened the titles and abstracts to arrive at a total of 121 articles, and 62 duplicates were excluded. Of the 83 articles, 30 did not meet the inclusion criteria for this review, thus 53 articles were eligible (Figure 1). Forty-one out of the fifty-three articles reported only one procedure, and twelve described more than one treatment. Only data from the four relevant categories were extrapolated and included in the pooled analysis. Thus, some studies have been replicated in the distribution among the four categories, taking into account that several authors described different procedures. Authors classified and staged MRONJ cases in all articles in accordance with the guidelines of the American Association of Oral and Maxillofacial Surgeons [27,28]; however, data regarding the extent of the disease was not always available.

Conservative surgery alone: 19 articles were included in this analysis. Even where different surgical approaches had been described, conservative surgery was always preceded by medical treatment (antiseptic mouthwash and systemic antibiotics). Nine out of the nineteen articles were case reports or case series, with a positive outcome in $100 \%$ of the treated patients ( $N=52$, cancer and osteoporotic patients), with a follow-up period of nearly twelve months. Of the remaining 10 articles $(\mathrm{N}=375$, stages 1, 2, 3 cancer and osteoporotic patients), the overall outcome was successful in the vast majority of cases, recording high success rates of $70 \%$ on average with a follow-up period of 9 months (Table 2).

Aggressive surgery alone: 7 articles were included in this analysis. Similarly, the practice of aggressive surgery was always preceded by medical treatment (antiseptic mouthwash and systemic antibiotics). Four of the seven articles were case reports or case series with positive outcomes in $100 \%$ of treated patients $(\mathrm{N}=25$, cancer and osteoporotic patients), with a follow-up period of nearly twelve months. In the 3 remaining articles ( $\mathrm{N}=245$ cancer and osteoporotic patients), the overall outcome was successful in the vast majority of cases, recording high success rates of $70 \%$ on average with a follow-up period of 12 months (Table 2). 

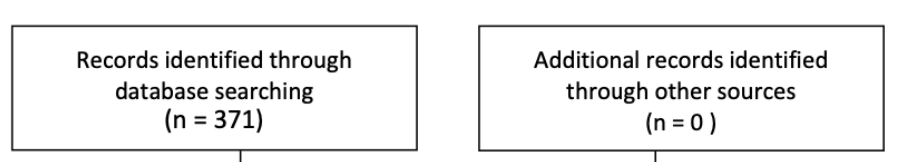
$(\mathrm{n}=371)$ through other sources<smiles>C1CCCCC1</smiles>

Records after duplicates removed $(n=62)$
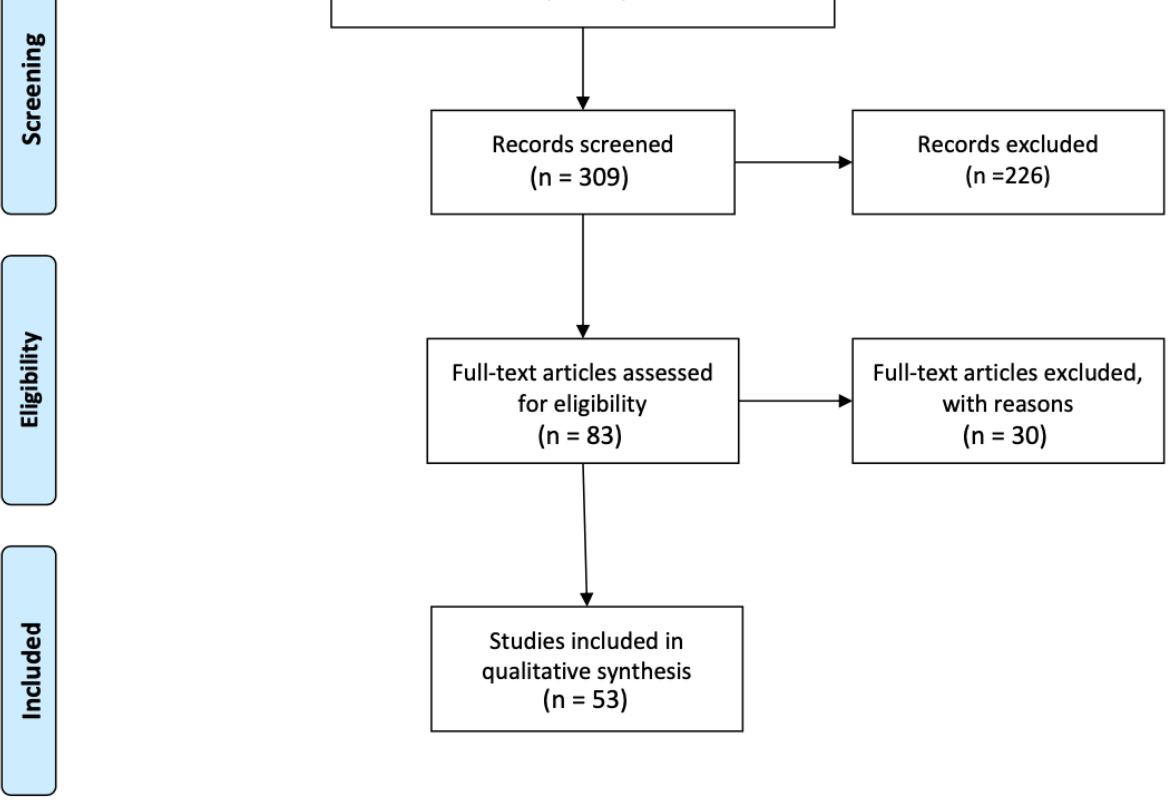
with reasons

From: Moher D, Liberati A, Tetzlaff J, Altman DG, The PRISMA Group (2009). Preferred Reporting /tems for Systematic Reviews and MetaAnalyses: The PRISMA Statement. PLoS Med 6(7): e1000097. doi:10.1371/journal.pmed1000097

$$
\text { For more information, visit www.prisma-statement.org. }
$$

Figure 1. A PRISMA flow chart of the pooled studies.

Conservative surgery plus non-invasive protocol (auxiliary treatment): 36 articles were included in this analysis, 22 of which were case reports or case series $(<10 \mathrm{pts})$ with a positive outcome in $100 \%$ of treated patients $(\mathrm{N}=51$, cancer and osteoporotic patients), with a follow-up period of nearly 11 months. In 15 articles $(\mathrm{N}=348$, cancer and osteoporotic patients), the overall outcome was successful in almost all cases, recording high success rates of $80 \%$ on average with a follow-up period of 8 months. All 36 articles were divided into 13 subcategories on the basis of different auxiliary treatments [29-59].

Aggressive surgery plus non-invasive procedures (auxiliary treatment): only two papers (case reports) discussed the results of aggressive surgery protocols with auxiliary treatment $[49,60]$.

Table 3 provides a comprehensive overview of these findings of the last categories.

Considering that only studies with $n>10$ patients were reviewed for the final pooled analysis, a total of 1137 patients were included in the study and assigned to one of the four categories according to their specific intervention. No significant differences between median age and sex were reported as the result of a comparison of the four groups. 
The overall 6-month total resolution rate (a) and the 6-month improvement rate (b) were: 74\% (CI 95\%; 64-83\%) and 87\% (CI 95\%; 78-94\%), respectively. The following was reported for $(\mathrm{a})$ :

- $\quad 80 \%$ (CI 95\%; 68-90\%) for invasive surgery alone (Figure 2a).

- $\quad 69 \%$ (95\% CI; 53-84\%) for invasive surgery plus non-invasive procedures (Figure $2 \mathrm{~b}$ ).

The following was reported for $(b)$ :

- $\quad 81 \%$ (CI 95\%; 67-92\%) for invasive surgery alone (Figure 3a).

- $\quad 92 \%$ (CI 95\%; 88-94\%) for invasive surgery plus non-invasive procedures (Figure 3b).

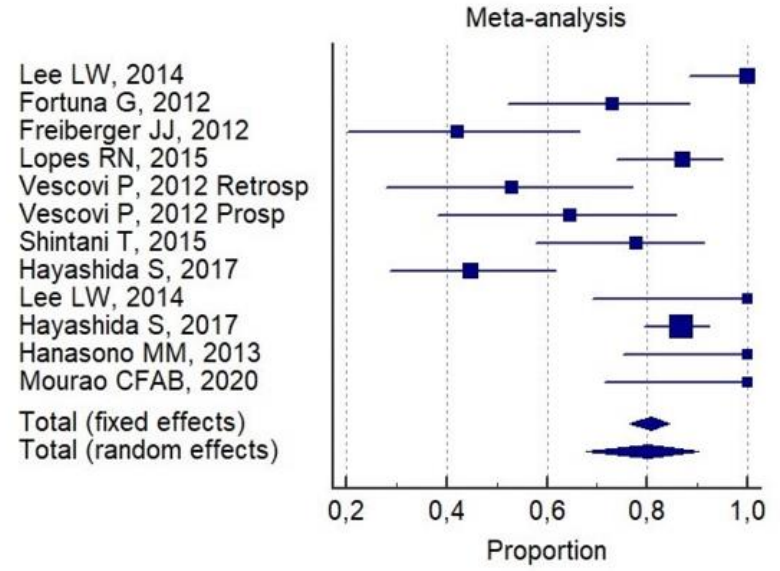

(a)

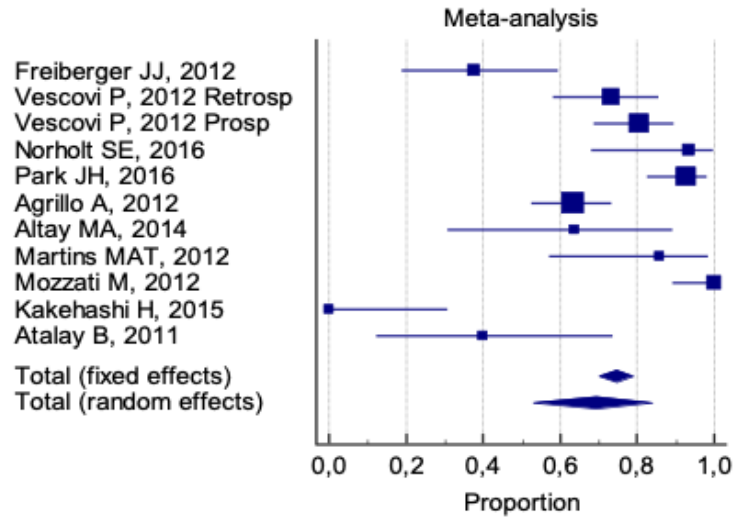

(b)

Figure 2. Forest plot results of pooled results about complete resolution in (a) invasive (conservative/aggressive) treatments, and (b) invasive (conservative/aggressive) treatments + non-invasive treatments.

Schubert M, 2012

Graziani F, 2012

Fortuna G, 2012

Freiberger JJ, 2012

Lopes RN, 2015

Vescovi P, 2012 Retrosp

Vescovi P, 2012 Prosp

Graziani F, 2012

Mourao CFAB, 2020

Total (fixed effects)

Total (random effects)

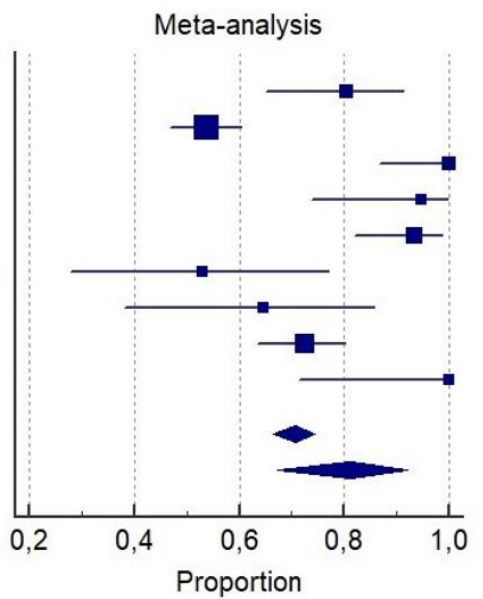

(a)

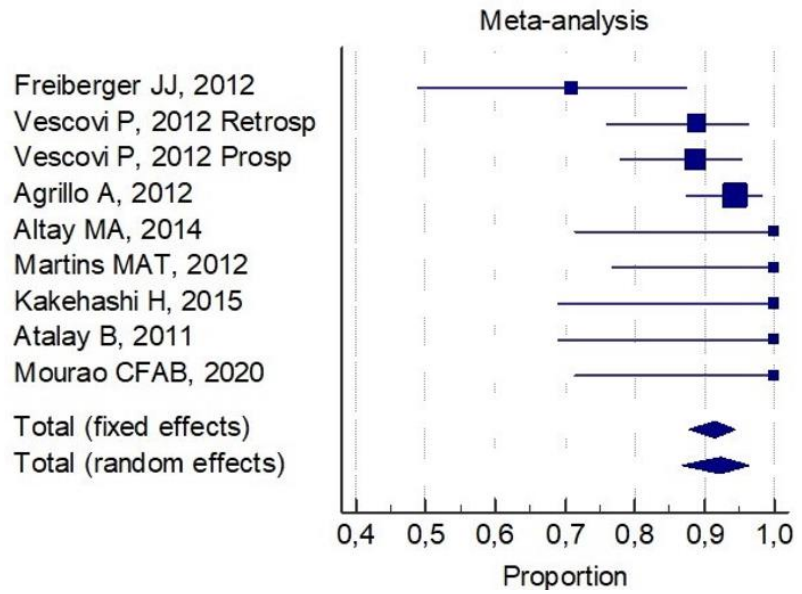

(b)

Figure 3. Forest plot results of pooled results about complete resolution in (a) invasive (conservative/aggressive) treatments, and (b) invasive (conservative/aggressive) plus non-invasive treatments.

Stratifying for each category of invasive procedures with respect to (a) and to (b), the data are collated in Table 4.

Of interest, a significant statistical difference was observed in the 6-month improvement rate, on comparing combined conservative surgery (mean $=91 \%$ ) versus only surgical (conservative alone and aggressive alone) techniques (mean $77 \%, p=0.05$ ). There was no significant difference for any group with respect to the 6-month total resolution rate $(82 \%$ versus $72 \%$, respectively). No reliable data were available for an analysis of aggressive surgery plus a non-invasive procedure with respect to all the selected indicators. 
Table 4. Stratification for each category of invasive procedures with respect to (a) and to (b).

\begin{tabular}{ccc}
\hline & $\begin{array}{c}\text { 6-Month Total Resolution Rate } \\
\text { (a) }\end{array}$ & 6-Month Improvement Rate (b) \\
\hline Conservative surgery alone & $67 \%(\mathrm{IC} 95 \% ; 50-83 \%)$ & $82 \%(\mathrm{IC} 95 \%, 65-95 \%)$ \\
Aggressive surgery alone & $93 \%(\mathrm{IC} 95 \% ; 82-99 \%)$ & $72 \%(\mathrm{IC} 95 \% ; 64-80 \%)$ \\
Conservative surgery plus non-invasive procedures & $75 \%(\mathrm{IC} 95 \% ; 60-87 \%)$ & $91 \%(\mathrm{IC} 95 \% ; 87-96 \%)$ \\
Aggressive plus non-invasive procedures & not assessable & not assessable \\
\hline
\end{tabular}

\section{Discussion}

The most profound effect of MRONJ in patients is the crippling nature of this disease with its negative impact on the quality of life. Thus, the challenge of the medical practitioner in treating these patients is undoubtedly to select the most appropriate medical protocols for maximizing a positive outcome for the patient.

Generally, the management of patients who are unable to achieve restitutio ad integrum (total recovery) will imply that the physician accompanies their patients on a journey of chronic illness. However, this may also include the pursuance of innovative research in adhering to appropriate protocols. Nowadays, it is possible for the symptoms of MRONJ to regress to a considerable degree or for the disease to partially heal in the absence of signs of phlogosis and demarcation of the necrotic process. All these features of healing can be considered as the primary goals for MRONJ management [61].

Unfortunately, there currently lacks consensus regarding the most appropriate treatment strategy for MRONJ. This may, in part, be due to the heterogeneity of MRONJ staging and available treatments; indeed, the majority of proposed protocols are surgical (conservative or aggressive). A conservative surgical approach can be deployed with the debridement of the superficial necrotic bone or by sequestrectomy, in addition to the use of systemic antibiotics and local antiseptic products [62-69]. An aggressive surgical approach will be adopted when conservative debridement has failed; in advanced cases of MRONJ, it is characterized by the partial or total resecting of the necrotic/non-necrotic tissue [70-72].

Two parameters, which are considered fundamental in deciding how to treat MRONJ, are staging and healing. A well-established staging system should be used to quantify the severity and extent of MRONJ and to guide decision-making [73]. Nowadays, AAOMS staging, as it is often termed, does not always satisfy the requisites for MRONJ treatment since it does not take into account the effective anatomical/radiological extent of the disease [28]. In this regard, Campisi et al. have recently highlighted this limitation, concluding that it may not always be effective and appropriate to propose MRONJ treatments based on the staging system [74]. The trials relating to their research, included in this systematic review, do not contain data stratified by disease stage, thereby precluding a pooled evaluation on this key topic. With the aim of facilitating reading, the authors of this paper have inserted the staging system used in every case series in order to complete the descriptive data (Tables 1 and 2). Of note, the choice of a given surgical protocol in some cases was found to be independent of the staging evaluation.

Healing is another pillar of MRONJ management. Based on an assessment of symptomatology, and clinical mucosal and radiological signs, specific terms have recently been proposed to improve the descriptions of treatment outcomes. Examples include: "resolved", "improving", "stable" and "progressive" [73]. Unfortunately, most academic papers have described the process of healing by merely referring to the integrity of mucosa as being seamless and symptomless, however without evaluating the underlying bone, from which the disease develops and recurs [75]. Furthermore, follow-up periods are often very short (approximately 6 months), and they exclude a recurrence at 6-12 months after the surgical procedure $[6,76]$. The analysis proposed herein has described healing as complete or partial as the authors have limited their descriptions to using undefined data.

Referring to the main results of this systematic analysis, high success rates $(70 \%$ on average) with a follow-up period of 9 months were recorded in almost all treated patients 
$(\mathrm{N}=375)$ who had been treated solely according to a conservative surgical approach. Approximately $70 \%$ of the 270 MRONJ patients, who had been treated solely with aggressive surgery and a follow-up of more than 12 months, also had a successful MRONJ outcome. Of this latter group, aggressive surgery alone was deployed in stage 1 of the disease $[23,65,77]$. This surgical choice is not supported by statements or guidelines, but it is left to the discretion of the surgeon; it does not, therefore, permit a reliable analysis of the results.

In a dissimilar manner, a non-invasive procedure (auxiliary therapy) was combined with conservative surgery, whilst there are fewer cases of aggressive surgery reported in the literature. When conservative surgery was combined with auxiliary therapy in the management of 401 patients, the optimum patient outcome was achieved by only considering the data of partial healing, and no differences have been reported in the literature when complete resolution (healing) was evaluated (Figures 2 and 3).

Auxiliary therapy consists of several agents with antiseptic, angiogenic and biomodulatory properties. Of these, platelet-rich fibrin (PRF) is a second-generation platelet concentrate. PRF membranes appear to be an easy and cheap alternative treatment with which to close exposed bone in MRONJ after surgical treatment [78-81]. They promote gingival healing by acting as a barrier membrane between the alveolar bone and the oral cavity, thereby accelerating physiologic wound healing and new bone formation. This minimizes recurrent infections and it prevents osteonecrotic lesions [34-36,38,50,82]. Moreover, leukocyte and platelet-rich fibrin (L-PRF and PRF, respectively) are determinant in immune regulation, which may be of importance in reducing tissue infection in the immediate postoperative period [83-85]. The association between surgery and blood components (such as platelet-rich-PRP or PRF or L-PRF) in the analysis presented in this paper has proved itself to be an effective treatment for the rapid closure of bone exposure and the formation of new gingival tissue in the absence of signs of phlogosis. With a success rate of $95 \%$, several authors have demonstrated that this association is effective on average over a 14-month follow-up period.

Another safe and effective treatment has also been described in the literature: PRF and PRP have been associated with laser phototherapy by virtue of the elevated microbicidal activity of the laser [16,43], making use of autologous bone marrow stem cells. This research has reported effective results by virtue of the osteogenic and chondrogenic potential of these agents (PRF and PRP), in addition to their capacity for vasculogenesis and angiogenesis [43]. De Santis et al [86] described two case reports treated with the debridement of the exposed necrotic bone followed by bone marrow stem cells injection: a positive follow up with no sing or symptoms in the necrotic area has been reported for the next 13 months.

Referring to the systematic review described herein, the associations between conservative surgery plus blood components, and laser or photodynamic therapy, appear to contribute much to: newly formed bone, the full coverage of bone tissue with healthy mucosa and the absence of symptoms and other signs of necrotic progression. This is due to the analgesic, anti-inflammatory and biomodulatory effects of blood components, and this protocol has been shown to be effective on average over a 6-month follow-up period with a success rate of $86 \%$.

The association of autologous bone marrow stem cells with conservative surgery and blood components has been reported only in one case study, with a success rate of $100 \%$ on average over a 6-month follow-up period. The CT scan revealed the diminution of osteolytic lesions with complete bone regeneration of the medial cortex of the lower jaw and a total resolution of symptoms.

An early resolution of MRONJ has been reported when combining conservative surgery and the use of bone morphogenetic protein (BMP-2) or SVC (stromal vascular fraction: a heterogeneous cell population containing mesenchymal stromal cells isolated by adipose tissue) and L-PRF: the addition of BMP to L-PRF stimulates soft tissue healing and bone remodeling, thereby promoting total mucosal coverage in the absence of signs of phlogosis and exposed bone, leading to a marked diminution of symptoms. Thus, patients 
requiring the continued administration of antiresorptive treatment may benefit from such a combined regimen [31,87].

Moving on to another study, Jung et al. [42] have proposed a concomitant administering of BMP-2 with teriparatide (TPTD) in order to maximize the regeneration of bone after surgery. In a synergistic manner, TPTD stimulates an anabolic effect by accelerating the osteoblastic differentiation of the BMP [88]. This result may prompt a paradigm shift in the treatment of MRONJ from resecting to regeneration. The association between BMP and TPTD had a success rate of $100 \%$ over a 3-month follow-up period. The addition of TPTD to BMP enhances remodeling and the formation of bone, thereby facilitating healing and the removal of necrotic bone. Many patients experience a complete resolution of their symptoms with no signs of phlogosis.

Low-level laser therapy (LLLT) has been used in treating MRONJ patients, together with an AF-guided surgical or a conventional surgical approach. It is considered by many as a safe and effective adjunct to the medical-surgical treatment of MRONJ lesions because it stimulates the regeneration and angiogenesis of soft tissues, thereby increasing the duration of the healing process. However, there still exists controversy regarding the physical and biological variables of LLLT, including: the type of laser, the frequency of the light pulse, output power, duration of application, fluence, and the distance of the source from the irradiated tissue [45-49]. The association of LLLT with surgery has demonstrated a success rate of $87 \%$ on average over a 12 -month follow-up period, with total mucosal healing in the absence of signs of infection or pain.

The use of surgery has also been associated with teriparatide (TPTD) treatment (prior to or after conventional surgical treatment) for MRONJ in osteoporotic patients. TPTD stimulates trabecular and cortical thickness, and trabecular connectivity and bone size bone formation by increasing osteoblast number and activity. Although successful results using TPTD treatment have been reported in the literature, its safety and efficacy are currently awaiting comprehensive evaluation. The treatment time during which it can be safely administered is strictly limited to less than 2 years in one lifespan [57-59]. A success rate of $83 \%$ on average over an 11-month follow-up period has been reported for the surgical treatment plus TPTD treatment (or vice versa) of MRONJ: any surgical wound completely healed with X-rays indicating stable alveolar bone. No inflammatory signs and symptoms have been reported to date.

Other protocols (for example, the use of ozone and hyperbaric oxygen (HBO)) have also been deployed and evaluated as a MRONJ surgical adjuvant treatment. Ozone has been used with different formulations (i.e., an oral irrigation of aqueous ozone, gas insufflation) and duration (prior to or after surgical curettage or sequestrectomy) by virtue of its positive features. These include: antimicrobial power, an enhancement of tissue oxygenation, an activation of the immune response, a stimulation of angiogenesis and fibroblast formation and analgesic agents $[51,55,56,89]$. In this review, the association between ozone and conservative surgery (or vice versa) demonstrated a success rate of $90-100 \%$ on average over a 22-month follow-up period. Complete mucosa healing was seen in the absence of symptoms such as pain and local inflammation.

As a pre-surgical treatment, $\mathrm{HBO}$ has successfully treated $\mathrm{MRONJ}$ lesions, thereby: improving the quality of life of afflicted patients [52-54], increasing wound healing, and reducing edema, inflammation and pain. $\mathrm{HBO}$ followed by surgical treatment had a success rate of $84 \%$ on average over an 18-month follow-up period, with: the complete healing of MRONJ lesion, total mucosal coverage, a cessation in the signs of infection and notable symptomatic relief.

\section{Conclusions}

The authors of this paper performed an evidence-based analysis which demonstrated the compelling and effective performance of non-invasive procedures, combined with conservative and aggressive surgery, in treating MRONJ patients. The data confirmed that partial and complete 6-month resolution rates ranged from $70 \%$ to $100 \%$. Of note, adjuvant 
therapy usually requires daily or weekly applications. Such a regular clinical practice permits the surgeon to constantly monitor the MRONJ lesion and to promptly modify treatment, where indicated. It is also hoped that many patients will be more inclined to maintain effective oral hygiene on account of their continual checkups. Moreover, an alleviation in symptoms has been achieved using appropriate treatments within a relatively short period of time and in the absence of negative events.

Many MRONJ patients can achieve total remission by means of aggressive surgical treatment, which is similar in mean duration to conservative surgery alone (9-12 months). This is notwithstanding other considerations, such as the patient suffering from a debilitating disease, the exacerbation of the quality of life with marked morbidity and, last but not least, challenging conditions for patients after aggressive surgery. These factors must be taken into account if patients also suffer from a significant systemic disease (e.g., metastatic patients) [41]. It might be opportune to highlight in cancer patients the appropriate choice of an MRONJ management protocol by conservative surgery with the addition of ozone [51], LLLT [45] or blood component + Nd:YAG [47] laser treatment. The analysis in this paper has demonstrated improved results in treating MRONJ with nearly total healing. Regrettably, there is a lack of reported data relating to the use of aggressive surgery plus auxiliary protocols, which would have been included in the pooled analysis.

Finally, some studies discussed in this paper confirmed an extended follow-up period for patients. This represents a key point in evaluating the healing of MRONJ, as has been previously highlighted by two Italian scientific bodies of oral pathology and medicine, and maxillofacial surgery: SIPMO and SICMF, respectively [74]. Their research has defined clinical and radiological MRONJ healing with a documented absence of symptoms and the clinical signs of MRONJ in a period of no less than 12 months $[1,3,90]$.

Despite the systematic nature of the analysis in this paper, there are limitations relating to: the non-randomized retrospective/prospective nature of the studies herein, the analysis of historical data, the heterogeneity of patients included in the study and a suitable definition of the endpoints being examined (the complete and partial resolutions of MRONJ symptoms).

To the best of our knowledge, the following regarding MRONJ treatment can be highlighted:

(1) A unanimous factual definition, including evaluation criteria (diagnosis and staging), is fundamental in assessing the efficacy of well-specified MRONJ treatment in order to facilitate a systematic analysis of the results of the research.

(2) The main positive outcomes of MRONJ treatment should be: the absence of symptoms, clinically active phlogosis and the obstacle of the relevant area of bone, as recognized on CT scans for a period of at least 12 months.

(3) Many in the field would say that the treatment of MRONJ is unquestionably related to its staging and the systemic status of the patient: cancer patients have often the worst quality of life, and aggressive surgery can exacerbate their condition.

(4) Conservative surgery combined with adjuvant procedures (i.e., ozone, LLLT or blood component + Nd:YAG laser treatment) can contribute to partial or total healing in all stages of MRONJ, with improved results and variables (from symptoms to clinical and radiological signs).

(5) Adjuvant therapy associated with surgery (conservative or aggressive) may be the future for MRONJ treatment. This combination could lead to the most positive results, but it is also of the utmost importance for conducting further effectively controlled studies in order to arrive at conclusive statements for the effective treatment of MRONJ.

Author Contributions: O.D.F.: Conceptualization, Methodology, Writing—original draft, Writingreview and editing; F.C.: Data curation, Methodology, Resources, Writing_original draft, Writingreview and editing; V.P.: Resources, Visualization; R.M.: Resources; C.D.G.: Data Curation, Visualization; A.B.: Visualization; V.F.: Visualization; P.T.: Supervision; G.P.: Validation, Supervision; G.C.: Validation, Supervision; A.G.: Data Curation, Methodology, Formal Analysis. All authors made substantial contributions to the study conception and design, and to the acquisition, analysis 
and interpretation of data. All were involved in drafting the manuscript or revising it critically for important intellectual content. All authors gave final approval of the version to be published, and agreed to be accountable for all aspects of the work in ensuring that questions relating to the accuracy or integrity of any part of the work will be appropriately investigated and resolved. All authors have read and agreed to the published version of the manuscript.

Funding: This research did not receive any specific grant from funding agencies in the public, commercial or not-for-profit sectors.

Institutional Review Board Statement: Not applicable.

Informed Consent Statement: Not applicable.

Data Availability Statement: No new data were created or analyzed in this study. Data sharing is not applicable to this article.

Acknowledgments: The present work was supported by Ministero dell'Istruzione dell'Università e della Ricerca (MIUR)_PON-AIM Line 1 (Id. AIM1892002) (recipient: R.M.).

Conflicts of Interest: The authors declare no relevant affiliations or financial involvement with any organization or entity with a financial interest in or financial conflict with the subject matter or materials discussed in the manuscript, apart from those disclosed.

\section{References}

1. Campisi, G.; Bedogni, A.; Fusco, V. Raccomandazioni Clinico-Terapeutiche Sull'osteonecrosi Delle Ossa Mascellari (ONJ) Farmaco-Relata e Sua Prevenzione; NDF, S., Ed.; Palermo University Press: Palermo, Italy, 2020.

2. Ruggiero, S.L.; Mehrotra, B.; Rosenberg, T.J.; Engroff, S.L. Osteonecrosis of the jaws associated with the use of bisphosphonates: A review of 63 cases. J. Oral Maxillofac. Surg. 2004, 62, 527-534. [CrossRef]

3. Khan, A.A.; Morrison, A.; Hanley, D.A.; Felsenberg, D.; McCauley, L.K.; O’Ryan, F.; Reid, I.; Ruggiero, S.L.; Taguchi, A.; Tetradis, S.; et al. Diagnosis and Management of Osteonecrosis of the Jaw: A Systematic Review and International Consensus. J. Bone Miner. Res. 2015, 30, 3-23. [CrossRef]

4. Kuroshima, S.; Sasaki, M.; Sawase, T. Medication-Related Osteonecrosis of the Jaw: A Literature Review. J. Oral Biosciences. Jpn. Assoc. Oral Biology 2019, 61, 99-104. [CrossRef] [PubMed]

5. Nicolatou-Galitis, O.; Schiødt, M.; Mendes, R.A.; Ripamonti, C.; Hope, S.; Drudge-Coates, L.; Niepel, D.; Wyngaert, T.V.D. Medication-related osteonecrosis of the jaw: Definition and best practice for prevention, diagnosis, and treatment. Oral Surg. Oral Med. Oral Pathol. Oral Radiol. 2019, 127, 117-135. [CrossRef]

6. Campisi, G.; Fedele, S.; Fusco, V.; Pizzo, G.; di Fede, O.; Bedogni, A. Epidemiology, clinical manifestations, risk reduction and treatment strategies of jaw osteonecrosis in cancer patients exposed to antiresorptive agents. Futur. Oncol. 2014, 10, 257-275. [CrossRef] [PubMed]

7. Schiodt, M.; Otto, S.; Fedele, S.; Bedogni, A.; Nicolatou-Galitis, O.; Guggenberger, R.; Herlofson, B.B.; Ristow, O.; Kofod, T. Workshop of European task force on medication-related osteonecrosis of the jaw-Current challenges. Oral Dis. 2019, 25, 1815-1821. [CrossRef]

8. Gómez-Moreno, G.; Arribas-Fernández, M.C.; Fernández-Guerrero, M.; Castro, A.B.; Aguilar-Salvatierra, A.; Guardia, J.; Botticelli, D.; Calvo-Guirado, J.L. Bisphosphonate-associated osteonecrosis of the jaw 2 years after teeth extractions: A case report solved with non-invasive treatment. Eur. Rev. Med. Pharmacol. Sci. 2014, 18, 1391-1397.

9. Moretti, F.; Pelliccioni, G.A.; Montebugnoli, L.; Marchetti, C. A prospective clinical trial for assessing the efficacy of a minimally invasive protocol in patients with bisphosphonate-associated osteonecrosis of the jaws. Oral Surg. Oral Med. Oral Pathol. Oral Radiol. Endodontology 2011, 112, 777-782. [CrossRef] [PubMed]

10. Bedogni, A.; Saia, G.; Bettini, G.; Tronchet, A.; Totola, A.; Bedogni, G.; Ferronato, G.; Nocini, P.F.; Blandamura, S. Long-term outcomes of surgical resection of the jaws in cancer patients with bisphosphonate-related osteonecrosis. Oral Oncol. 2011, 47, 420-424. [CrossRef] [PubMed]

11. Beth-Tasdogan, N.H.; Mayer, B.; Hussein, H.; Zolk, O. Interventions for managing medication-related osteonecrosis of the jaw. Cochrane Database Syst. Rev. 2017, 10, CD012432. [CrossRef]

12. Laimer, J.; Steinmassl, O.; Hechenberger, M.; Rasse, M.; Pikula, R.; Bruckmoser, E. Intraoral Vacuum-Assisted Closure Therapy-A Pilot Study in Medication-Related Osteonecrosis of the Jaw. J. Oral Maxillofac. Surg. 2017, 75, 2154-2161. [CrossRef]

13. Owosho, A.A.; Estilo, C.L.; Huryn, J.M.; Yom, S.K. Pentoxifylline and tocopherol in the management of cancer patients with medication-related osteonecrosis of the jaw: An observational retrospective study of initial case series. Oral Surg. Oral Med. Oral Pathol. Oral Radiol. 2016, 122, 455-459. [CrossRef]

14. Magremanne, M.; Reychler, H. Pentoxifylline and Tocopherol in the Treatment of Yearly Zoledronic Acid-Related Osteonecrosis of the Jaw in a Corticosteroid-Induced Osteoporosis. J. Oral Maxillofac. Surg. 2014, 72, 334-337. [CrossRef] [PubMed] 
15. Porcaro, G.; Amosso, E.; Scarpella, R.; Carini, F. Doxycycline fluorescence-guided Er:YAG laser ablation combined with Nd:YAG/diode laser biostimulation for treating bisphosphonate-related osteonecrosis of the jaw. Oral Surg. Oral Med. Oral Pathol. Oral Radiol. 2015, 119, e6-e12. [CrossRef] [PubMed]

16. Luomanen, M.; Alaluusua, S. Treatment of bisphosphonate-induced osteonecrosis of the jaws with Nd:YAG laser biostimulation. Lasers Med. Sci. 2011, 27, 251-255. [CrossRef] [PubMed]

17. Heggendorn, F.L.; Leite, T.C.; Cunha, K.S.G.; Júnior, A.S.; Gonçalves, L.S.; Da Costa, K.B.F.F.; Dias, E.P. Bisphosphonate-related osteonecrosis of the jaws: Report of a case using conservative protocol. Spéc. Care Dent. 2016, 36, 43-47. [CrossRef] [PubMed]

18. Yoshiga, D.; Yamashita, Y.; Nakamichi, I.; Tanaka, T.; Yamauchi, K.; Yamamoto, N.; Nogami, S.; Kaneuji, T.; Mitsugi, S.; Sakurai, T.; et al. Weekly teriparatide injections successfully treated advanced bisphosphonate-related osteonecrosis of the jaws. Osteoporos. Int. 2013, 24, 2365-2369. [CrossRef] [PubMed]

19. Ohbayashi, Y.; Miyake, M.; Sawai, F.; Minami, Y.; Iwasaki, A.; Matsui, Y. Adjunct teriparatide therapy with monitoring of bone turnover markers and bone scintigraphy for bisphosphonate-related osteonecrosis of the jaw. Oral Surg. Oral Med. Oral Pathol. Oral Radiol. 2013, 115, e31-e37. [CrossRef]

20. Yamachika, E.; Matsubara, M.; Ikeda, A.; Matsumura, T.; Moritani, N.; Iida, S. Treatment of Osteonecrosis of the Jaw. J. Craniofacial Surg. 2015, 26, e575-e577. [CrossRef] [PubMed]

21. Thumbigere-Math, V.; Michalowicz, B.S.; Hodges, J.S.; Tsai, M.L.; Swenson, K.K.; Rockwell, L.; Gopalakrishnan, R. Periodontal disease as a risk factor for bisphosphonate-related osteonecrosis of the jaw. J. Periodontol. 2014, 85, 226-233. [CrossRef]

22. Mauceri, R.; Panzarella, V.; Maniscalco, L.; Bedogni, A.; Licata, M.E.; Albanese, A.; Toia, F.; Cumbo, E.M.G.; Mazzola, G.; Di Fede, O.; et al. Conservative Surgical Treatment of Bisphosphonate-Related Osteonecrosis of the Jaw with Er,Cr:YSGG Laser and Platelet-Rich Plasma: A Longitudinal Study. BioMed Res. Int. 2018, 2018, 1-10. [CrossRef]

23. Hayashida, S.; Soutome, S.; Yanamoto, S.; Fujita, S.; Hasegawa, T.; Komori, T.; Kojima, Y.; Miyamoto, H.; Shibuya, Y.; Ueda, N.; et al. Evaluation of the Treatment Strategies for Medication-Related Osteonecrosis of the Jaws (MRONJ) and the Factors Affecting Treatment Outcome: A Multicenter Retrospective Study with Propensity Score Matching Analysis. J. Bone Min. Res. 2017, 32, 2022-2029. [CrossRef] [PubMed]

24. Aljohani, S.; Troeltzsch, M.; Hafner, S.; Kaeppler, G.; Mast, G.; Otto, S. Surgical treatment of medication-related osteonecrosis of the upper jaw: Case series. Oral Dis. 2019, 25, 497-507. [CrossRef]

25. Schiodt, M.; Vadhan-Raj, S.; Chambers, M.S.; Nicolatou-Galitis, O.; Politis, C.; Coropciuc, R.; Fedele, S.; Jandial, D.; Zhang, J.; $\mathrm{Ma}, \mathrm{H}$; ; et al. A multicenter case registry study on medication-related osteonecrosis of the jaw in patients with advanced cancer. Support. Care Cancer 2018, 26, 1905-1915. [CrossRef]

26. Ristow, O.; Rückschloß, T.; Müller, M.; Berger, M.; Kargus, S.; Pautke, C.; Engel, M.; Hoffmann, J.; Freudlsperger, C. Is the conservative non-surgical management of medication-related osteonecrosis of the jaw an appropriate treatment option for early stages? A long-term single-center cohort study. J. Cranio-Maxillofacial Surg. 2019, 47, 491-499. [CrossRef]

27. Ruggiero, S.L.; Dodson, T.B.; Assael, L.A.; Landesberg, R.; Marx, R.E.; Mehrotra, B. American Association of Oral and Maxillofacial Surgeons Position Paper on Bisphosphonate-Related Osteonecrosis of the Jaws-2009 Update. J. Oral Maxillofac. Surg. 2009, 67, 2-12. [CrossRef]

28. Ruggiero, S.L.; Dodson, T.B.; Fantasia, J.; Goodday, R.; Aghaloo, T.; Mehrotra, B.; O’Ryan, F. American Association of Oral and Maxillofacial Surgeons Position Paper on Medication-Related Osteonecrosis of the Jaw-2014 Update. J. Oral Maxillofac. Surg. 2014, 72, 1938-1956. [CrossRef]

29. Mozzati, M.; Gallesio, G.; Arata, V.; Pol, R.; Scoletta, M. Platelet-rich therapies in the treatment of intravenous bisphosphonaterelated osteonecrosis of the jaw: A report of 32 cases. Oral Oncol. 2012, 48, 469-474. [CrossRef]

30. Bocanegra-Pérez, S.; Vicente-Barrero, M.; Knezevic, M.; Castellano-Navarro, J.; Bocanegra, E.R.; Rodríguez-Millares, J.; PérezPlasencia, D.; Ramos-Macías, A. Use of platelet-rich plasma in the treatment of bisphosphonate-related osteonecrosis of the jaw. Int. J. Oral Maxillofac. Surg. 2012, 41, 1410-1415. [CrossRef] [PubMed]

31. Park, J.-H.; Kim, J.-W.; Kim, S.-J. Does the Addition of Bone Morphogenetic Protein 2 to Platelet-Rich Fibrin Improve Healing After Treatment for Medication-Related Osteonecrosis of the Jaw? J. Oral Maxillofac. Surg. 2017, 75, 1176-1184. [CrossRef] [PubMed]

32. Anitua, E.; Begoña, L.; Orive, G. Treatment of hemimandibular paresthesia in a patient with bisphosphonate-related osteonecrosis of the jaw (BRONJ) by combining surgical resection and PRGF-Endoret. Br. J. Oral Maxillofac. Surg. 2013, 51, e272-e274. [CrossRef]

33. Pelaz, A.; Junquera, L.; Gallego, L.; Garcia-Consuegra, L.; Gomez, C. Alternative treatments for oral bisphosphonate-related osteonecrosis of the jaws: A pilot study comparing fibrin rich in growth factors and teriparatide. Med. Oral Patol. Oral Cir. Bucal 2014, 19, e320-e326. [CrossRef] [PubMed]

34. Dincă, O.; Zurac, S.; Stăniceanu, F.; Bucur, M.B.; Bodnar, D.C.; Vlădan, C.; Bucur, A. Clinical and histopathological studies using fibrin-rich plasma in the treatment of bisphosphonate-related osteonecrosis of the jaw. Rom. J. Morphol. Embryol. Rev. Roum. Morphol. Embryol. 2014, 55, 961-964.

35. Nørholt, S.; Hartlev, J. Surgical treatment of osteonecrosis of the jaw with the use of platelet-rich fibrin: A prospective study of 15 patients. Int. J. Oral Maxillofac. Surg. 2016, 45, 1256-1260. [CrossRef]

36. Soydan, S.S.; Uckan, S. Management of Bisphosphonate-Related Osteonecrosis of the Jaw With a Platelet-Rich Fibrin Membrane: Technical Report. J. Oral Maxillofac. Surg. 2014, 72, 322-326. [CrossRef] [PubMed] 
37. Tsai, L.-L.; Huang, Y.-F.; Chang, Y.-C. Treatment of bisphosphonate-related osteonecrosis of the jaw with platelet-rich fibrin. J. Formos. Med. Assoc. 2016, 115, 585-586. [CrossRef]

38. Maluf, G.; de Pinho, M.C.; da Cunha SR de, B.; Santos PS da, S.; Fregnani, E.R. Surgery combined with lprf in denosumab osteonecrosis of the jaw: Case report. Braz. Dent. J. 2016, 27, 353-358. [CrossRef]

39. Gönen, Z.B.; Asan, C.Y. Treatment of bisphosphonate-related osteonecrosis of the jaw using platelet-rich fibrin. CRANIO 2016, 35 , 332-336. [CrossRef]

40. de Castro, M.S.; Ribeiro, N.V.; de Carli, M.L.; Pereira, A.A.C.; Sperandio, F.F.; Hanemann, J.A.C. Photodynamically dealing with bisphosphonate-related osteonecrosis of the jaw: Successful case reports. Photodiagnosis Photodyn Ther. 2016, 16, 72-75. [CrossRef]

41. Lee, J.-J.; Cheng, S.-J.; Jeng, J.-H.; Chiang, C.-P.; Lau, H.-P.; Kok, S.-H. Successful treatment of advanced bisphosphonate-related osteonecrosis of the mandible with adjunctive teriparatide therapy. Head Neck 2010, 33, 1366-1371. [CrossRef] [PubMed]

42. Jung, J.; Yoo, H.-Y.; Kim, G.-T.; Lee, J.-W.; Lee, Y.-A.; Kim, D.-Y.; Kwon, Y.-D. Short-Term Teriparatide and Recombinant Human Bone Morphogenetic Protein-2 for Regenerative Approach to Medication-Related Osteonecrosis of the Jaw: A Preliminary Study. J. Bone Miner. Res. 2017, 32, 2445-2452. [CrossRef]

43. Gonzálvez-García, M.; Rodríguez-Lozano, F.J.; Villanueva, V.; Segarra-Fenoll, D.; Rodriguez-Gonzalez, M.A.; Oñate-Sánchez, R.; Blanquer, M.; Moraleda, J.M. Cell Therapy in Bisphosphonate-Related Osteonecrosis of the Jaw. J. Craniofacial Surg. 2013, 24, e226-e228. [CrossRef]

44. Da Guarda, M.G.; Paraguassú, G.M.; Cerqueira, N.S.; Cury, P.R.; Farias, J.G.; Ramalho, L.M.P. Laser GaAlAs ( $\lambda 860 \mathrm{~nm})$ photobiomodulation for the treatment of bisphosphonate-induced osteonecrosis of the jaw. Photomed. Laser Surg. 2012, 30, $293-297$. [CrossRef]

45. Altay, M.A.; Tasar, F.; Tosun, E.; Kan, B. Low-Level Laser Therapy Supported Surgical Treatment of Bisphosphonate Related Osteonecrosis of Jaws: A Retrospective Analysis of 11 Cases. Photomed. Laser Surg. 2014, 32, 468-475. [CrossRef]

46. Atalay, B.; Yalcin, S.; Emes, Y.; Aktas, I.; Aybar, B.; Issever, H.; Mandel, N.M.; Cetin, O.; Oncu, B. Bisphosphonate-related osteonecrosis: Laser-assisted surgical treatment or conventional surgery? Lasers Med. Sci. 2011, 26, 815-823. [CrossRef]

47. Vescovi, P.; Manfredi, M.; Merigo, E.; Guidotti, R.; Meleti, M.; Pedrazzi, G.; Fornaini, C.; Bonanini, M.; Ferri, T.; Nammour, S. Early Surgical Laser-Assisted Management of Bisphosphonate-Related Osteonecrosis of the Jaws (BRONJ): A Retrospective Analysis of 101 Treated Sites with Long-Term Follow-Up. Photomed. Laser Surg. 2012, 30, 5-13. [CrossRef] [PubMed]

48. Vescovi, P.; Merigo, E.; Meleti, M.; Manfredi, M.; Guidotti, R.; Nammour, S. Bisphosphonates-related osteonecrosis of the jaws: A concise review of the literature and a report of a single-centre experience with 151 patients. J. Oral Pathol. Med. 2011, 41, 214-221. [CrossRef] [PubMed]

49. Vescovi, P.; Giovannacci, I.; Otto, S.; Manfredi, M.; Merigo, E.; Fornaini, C.; Nammour, S.; Meleti, M. Medication-Related Osteonecrosis of the Jaw: An Autofluorescence-Guided Surgical Approach Performed with Er:YAG Laser. Photomed. Laser Surg. 2015, 33, 437-442. [CrossRef] [PubMed]

50. Martins, M.A.T.; Martins, M.D.; LaScala, C.A.; Curi, M.M.; Migliorati, C.A.; Tenis, C.A.; Marques, M. Association of laser phototherapy with PRP improves healing of bisphosphonate-related osteonecrosis of the jaws in cancer patients: A preliminary study. Oral Oncol. 2012, 48, 79-84. [CrossRef]

51. Agrillo, A.; Filiaci, F.; Ramieri, V.; Riccardi, E.; Quarato, D.; Rinna, C.; Gennaro, P.; Cascino, F.; Mitro, V.; Ungari, C. Bisphosphonaterelated osteonecrosis of the jaw (BRONJ): 5 year experience in the treatment of 131 cases with ozone therapy. Eur. Rev. Med. Pharmacol. Sci. 2012, 16, 1741-1747.

52. Al-Zoman, K.H.; Albazie, S.; Robert, A.A.; Baskaradoss, J.K.; Alsuwyed, A.S.; Ciancio, S.; Al-Mubarak, S. Surgical management of bisphosphonate-related osteonecrosis of the jaw: Report of three cases. J. Palliat. Care 2013, 29, 52-57. [CrossRef] [PubMed]

53. Fatema, C.N.; Sato, J.; Yamazaki, Y.; Hata, H.; Hattori, N.; Shiga, T.; Tamaki, N.; Kitagawa, Y. FDG-PET may predict the effectiveness of hyperbaric oxygen therapy in a patient with bisphosphonate-related osteonecrosis of the jaw: Report of a case. Odontology 2013, 103, 105-108. [CrossRef]

54. Freiberger, J.J.; Padilla-Burgos, R.; McGraw, T.; Suliman, H.B.; Kraft, K.H.; Stolp, B.W.; Moon, R.E.; Piantadosi, C.A. What Is the Role of Hyperbaric Oxygen in the Management of Bisphosphonate-Related Osteonecrosis of the Jaw: A Randomized Controlled Trial of Hyperbaric Oxygen as an Adjunct to Surgery and Antibiotics. J. Oral Maxillofac. Surg. 2012, 70, 1573-1583. [CrossRef]

55. Ripamonti, C.; Maniezzo, M.; Boldini, S.; Pessi, M.; Mariani, L.; Cislaghi, E. Efficacy and tolerability of medical ozone gas insufflations in patients with osteonecrosis of the jaw treated with bisphosphonates-Preliminary data: Medical ozone gas insufflation in treating ONJ lesions. J. Bone Oncol. 2012, 1, 81-87. [CrossRef]

56. Brozoski, M.A.; Traina, A.A.; Deboni, M.C.Z.; Marques, M.M.; Naclério-Homem M da, G. Bisphosphonate-related osteonecrosis of the jaw. Rev. Bras. Reumatol. 2020, 52, 265-270. [CrossRef]

57. Kwon, Y.-D.; Lee, D.-W.; Choi, B.-J.; Lee, J.-W.; Kim, D.-Y. Short-term teriparatide therapy as an adjunctive modality for bisphosphonate-related osteonecrosis of the jaws. Osteoporos. Int. 2012, 23, 2721-2725. [CrossRef]

58. Doh, R.-M.; Park, H.-J.; Rhee, Y.; Kim, H.S.; Huh, J.; Park, W. Teriparatide Therapy for Bisphosphonate-Related Osteonecrosis of the Jaw Associated With Dental Implants. Implant. Dent. 2015, 24, 222-226. [CrossRef]

59. Kakehashi, H.; Ando, T.; Minamizato, T.; Nakatani, Y.; Kawasaki, T.; Ikeda, H.; Kuroshima, S.; Kawakami, A.; Asahina, I. Administration of teriparatide improves the symptoms of advanced bisphosphonate-related osteonecrosis of the jaw: Preliminary findings. Int. J. Oral Maxillofac. Surg. 2015, 44, 1558-1564. [CrossRef] [PubMed] 
60. Rahim, I.; Salt, S.; Heliotis, M. Successful long-term mandibular reconstruction and rehabilitation using non-vascularised autologous bone graft and recombinant human BMP-7 with subsequent endosseous implant in a patient with bisphosphonaterelated osteonecrosis of the jaw. Br. J. Oral Maxillofac. Surg. 2015, 53, 870-874. [CrossRef]

61. Montebugnoli, L.; Felicetti, L.; Gissi, D.B.; Pizzigallo, A.; Pelliccioni, G.A.; Marchetti, C. Biphosphonate-associated osteonecrosis can be controlled by nonsurgical management. Oral Surg. Oral Med. Oral Pathol. Oral Radiol. Endodontology 2007, 104, $473-477$. [CrossRef] [PubMed]

62. de Souza Póvoa, R.C.; Marlierè, D.A.A.; da Silveira, H.M.; Pires, F.R. Denosumab-related osteonecrosis of the jaws: Successful management with a conservative surgical approach. Spec. Care Dent. 2016, 36, 231-236. [CrossRef]

63. Ribeiro, N.R.B.; Silva, L.D.F.; Santana, D.M.; Nogueira, R.L.M. Bisphosphonate-Related Osteonecrosis of the Jaw After Tooth Extraction. J. Craniofacial Surg. 2015, 26, e606-e608. [CrossRef]

64. de Souza Faloni, A.P.; Queiroz, T.P.; Lia RC, C.; Cerri, P.S.; Margonar, R.; de Souza Rastelli, A.N.; Marcantonio, E. Accurate approach in the treatment of oral bisphosphonate-related jaw osteonecrosis. J. Craniofac. Surg. 2011, 22, 2185-2190. [CrossRef]

65. Lee, L.-W.; Hsiao, S.-H.; Chen, L.-K. Clinical treatment outcomes for 40 patients with bisphosphonates-related osteonecrosis of the jaws. J. Formos. Med. Assoc. 2014, 113, 166-172. [CrossRef]

66. Graziani, F.; Vescovi, P.; Campisi, G.; Favia, G.; Gabriele, M.; Gaeta, G.M.; Gennai, S.; Goia, F.; Miccoli, M.; Peluso, F.; et al. Resective Surgical Approach Shows a High Performance in the Management of Advanced Cases of Bisphosphonate-Related Osteonecrosis of the Jaws: A Retrospective Survey of 347 Cases. J. Oral Maxillofac. Surg. 2012, 70, 2501-2507. [CrossRef]

67. Duarte, L.F.M.; Alonso, K.; Basso, E.C.; Dib, L.L. Surgical Treatment of Bisphosphonate-Related Osteonecrosis of the Jaws with the Use of Buccal Fat Pad: Case Report. Braz. Dent. J. 2015, 26, 317-320. [CrossRef]

68. Berrone, M.; Florindi, F.U.; Carbone, V.; Aldiano, C.; Pentenero, M. Stage 3 Medication-Related Osteonecrosis of the Posterior Maxilla: Surgical Treatment Using a Pedicled Buccal Fat Pad Flap: Case Reports. J. Oral Maxillofac. Surg. 2015, 73, 2082-2086. [CrossRef]

69. Lopes, R.N.; Rabelo, G.; Rocha, A.C.; Carvalho, P.A.G.; Alves, F.A. Surgical Therapy for Bisphosphonate-Related Osteonecrosis of the Jaw: Six-Year Experience of a Single Institution. J. Oral Maxillofac. Surg. 2015, 73, 1288-1295. [CrossRef]

70. Hewson, I.; Syme, D.; Bruscino-Raiola, F. Radical surgical treatment of bisphosphonate related osteonecrosis of the jaw. Aust. Dent. J. 2012, 57, 227-230. [CrossRef] [PubMed]

71. Ghazali, N.; Collyer, J.; Tighe, J. Hemimandibulectomy and vascularized fibula flap in bisphosphonate-induced mandibular osteonecrosis with polycythaemia rubra vera. Int. J. Oral Maxillofac. Surg. 2013, 42, 120-123. [CrossRef]

72. Hanasono, M.M.; Militsakh, O.; Richmon, J.D.; Rosenthal, E.L.; Wax, M.K. Mandibulectomy and Free Flap Reconstruction for Bisphosphonate-Related Osteonecrosis of the Jaws. JAMA Otolaryngol. Neck Surg. 2013, 139, 1135-1142. [CrossRef]

73. Yarom, N.; Shapiro, C.L.; Peterson, D.E.; Van Poznak, C.H.; Bohlke, K.; Ruggiero, S.L.; Migliorati, C.A.; Khan, A.; Morrison, A.; Anderson, H.; et al. Medication-related osteonecrosis of the jaw: MASCC/ISOO/ASCO clinical practice guideline. J. Clin. Oncol. 2019, 37, 2270-2290. [CrossRef]

74. Campisi, G.; Mauceri, R.; Bertoldo, F.; Bettini, G.; Biasotto, M.; Colella, G.; Consolo, U.; Di Fede, O.; Favia, G.; Fusco, V.; et al. Medication-Related Osteonecrosis of Jaws (MRONJ) Prevention and Diagnosis: Italian Consensus Update 2020. Int. J. Environ. Res. Public Health 2020, 17, 5998. [CrossRef]

75. Wilde, F.; Heufelder, M.; Winter, K.; Hendricks, J.; Frerich, B.; Schramm, A.; Hemprich, A. The role of surgical therapy in the management of intravenous bisphosphonates-related osteonecrosis of the jaw. Oral Surg. Oral Med. Oral Pathol. Oral Radiol. Endodontology 2011, 111, 153-163. [CrossRef]

76. Carlson, E.R.; Basile, J.D. The Role of Surgical Resection in the Management of Bisphosphonate-Related Osteonecrosis of the Jaws. J. Oral Maxillofac. Surg. 2009, 67, 85-95. [CrossRef]

77. Shintani, T.; Hayashido, Y.; Mukasa, H.; Akagi, E.; Hoshino, M.; Ishida, Y.; Hamana, T.; Okamoto, K.; Kanda, T.; Koizumi, K.; et al. Comparison of the prognosis of bisphosphonate-related osteonecrosis of the jaw caused by oral and intravenous bisphosphonates. Int. J. Oral Maxillofac. Surg. 2015, 44, 840-844. [CrossRef]

78. Choukroun, J.; Adda, F.; Schoeffler, C.; Vervelle, A. Une opportunité en paro-implantologie: Le PRF. Implantodontie 2001, 42 , e62.

79. Dohan, D.M.; Choukroun, J.; Diss, A.; Dohan, S.L.; Dohan, A.; Mouhyi, J.; Gogly, B. Platelet-rich fibrin (PRF): A second-generation platelet concentrate. Part II: Platelet-related biologic features. Oral Surg. Oral Med. Oral Pathol. Oral Radiol. Endodontology 2006, 101, e45-e50. [CrossRef]

80. Mourão, C.F.D.A.B.; Calasans-Maia, M.D.; Del Fabbro, M.; Vieira, F.L.D.; Machado, R.; Capella, R.; Miron, R.J.; Alves, G.G. The use of Platelet-rich Fibrin in the management of medication-related osteonecrosis of the jaw: A case series. J. Stomatol. Oral Maxillofac. Surg. 2020, 121, 84-89. [CrossRef]

81. Antonelli, A.; Giudice, A.; Muraca, D.; Fortunato, L. Usefulness of advanced-platelet rich fibrin (A-PRF) and injectable-platelet rich fibrin (i-PRF) in the management of a massive medication-related osteonecrosis of the jaw (MRONJ): A 5-years follow-up case report. Indian J. Dent. Res. 2020, 31, 813-818. [CrossRef]

82. Schubert, M.; Klatte, I.; Linek, W.; Müller, B.; Döring, K.; Eckelt, U.; Hemprich, A.; Berger, U.; Hendricks, J. The Saxon Bisphosphonate Register-Therapy and prevention of bisphosphonate-related osteonecrosis of the jaws. Oral Oncol. 2012, 48, 349-354. [CrossRef] [PubMed] 
83. Pechalova, P.; Bakardjiev, A.; Zaprianov, Z.; Vladimirov, B.; Poriazova, E.; Zheleva, A.; Hadjigeorgiev, G.; Goranova-Marinova, V.; Goranov, S. Bisphosphonate-associated osteonecrosis of the jaws-report of three cases in bulgaria and review of the literature. Acta Clin. Croatica. 2011, 50, 273-279.

84. Gallego, L.; Junquera, L.; Pelaz, A.; Hernando, J.; Megias, J. The use of pedicled buccal fat pad combined with sequestrectomy in bisphosphonate-related osteonecrosis of the maxilla. Med. Oral Patol. Oral Cir. Bucal 2012, 17, e236-e241. [CrossRef]

85. Fortuna, G.; Ruoppo, E.; Pollio, A.; Aria, M.; Adamo, D.; Leuci, S.; Orabona, G.D.; Mignogna, M.D. Multiple myeloma vs. breast cancer patients with bisphosphonates-related osteonecrosis of the jaws: A comparative analysis of response to treatment and predictors of outcome. J. Oral Pathol. Med. 2011, 41, 222-228. [CrossRef]

86. De Santis, G.C.; Macedo, L.; Orellana, M.D.; Innocentini, L.M.A.R.; Ferrari, T.C.; Ricz, H.M.A.; Caruso, S.R.; Fernandes, T.R.; Covas, D.T. Mesenchymal stromal cells administration for osteonecrosis of the jaw caused by bisphosphonate: Report of two cases. Acta Oncol. 2020, 59, 789-792. [CrossRef]

87. Bouland, C.; Meuleman, N.; Widelec, J.; Keiani-Mothlagh, K.; Voisin, C.; Lagneaux, L.; Philippart, P. Case reports of medicationrelated osteonecrosis of the jaw (MRONJ) treated with uncultured stromal vascular fraction and L-PRF. J. Stomatol. Oral Maxillofac. Surg. 2021, 122, 212-218. [CrossRef] [PubMed]

88. Marconcini, S.; Denaro, M.; Cosola, S.; Gabriele, M.; Toti, P.; Mijiritsky, E.; Proietti, A.; Basolo, F.; Giammarinaro, E.; Covani, U. Myofibroblast Gene Expression Profile after Tooth Extraction in the Rabbit. Materials 2019, 12, 3697. [CrossRef] [PubMed]

89. Marconcini, S.; Giammarinaro, E.; Cosola, S.; Genovesi, A.M.; Covani, U. Mandibular Osteonecrosis Associated with Antacid Therapy (Esomeprazole). Eur. J. Case Rep. Intern. Med. 2019, 6. [CrossRef]

90. Bedogni, A.; Fedele, S.; Bedogni, G.; Scoletta, M.; Favia, G.; Colella, G.; Agrillo, A.; Bettini, G.; Di Fede, O.; Oteri, G.; et al. Staging of osteonecrosis of the jaw requires computed tomography for accurate definition of the extent of bony disease. Br. J. Oral Maxillofac. Surg. 2014, 52, 603-608. [CrossRef] 